Portland State University

PDXScholar

\title{
Asymptotic and Numerical Techniques for Resonances of Thin Photonic Structures
}

\author{
Jay Gopalakrishnan \\ Portland State University, gjay@pdx.edu \\ Shari Moskow \\ Drexel University \\ Fadil Santosa \\ University of Minnesota - Twin Cities
}

Follow this and additional works at: https://pdxscholar.library.pdx.edu/mth_fac

Part of the Applied Mathematics Commons, and the Mathematics Commons Let us know how access to this document benefits you.

\section{Citation Details}

Gopalakrishnan, Jay; Moskow, Shari; and Santosa, Fadil, "Asymptotic and Numerical Techniques for Resonances of Thin Photonic Structures" (2008). Mathematics and Statistics Faculty Publications and Presentations. 62.

https://pdxscholar.library.pdx.edu/mth_fac/62

This Post-Print is brought to you for free and open access. It has been accepted for inclusion in Mathematics and Statistics Faculty Publications and Presentations by an authorized administrator of PDXScholar. Please contact us if we can make this document more accessible: pdxscholar@pdx.edu. 


\title{
ASYMPTOTIC AND NUMERICAL TECHNIQUES FOR RESONANCES OF THIN PHOTONIC STRUCTURES.*
}

\author{
J. GOPALAKRISHNAN ${ }^{\dagger}$, S. MOSKOW ${ }^{\ddagger}$, AND F. SANTOSA ${ }^{\S}$
}

\begin{abstract}
We consider the problem of calculating resonance frequencies and radiative losses of an optical resonator. The optical resonator is in the form of a thin membrane with variable dielectric properties. This work provides two very different approaches for doing such calculations. The first is an asymptotic method which exploits the small thickness and high index of the membrane. We derive a limiting resonance problem as the thickness goes to zero, and for the case of a simple resonance, find a first order correction. The limiting problem and the correction are in one less space dimension, which can make the approach very efficient. Convergence estimates are proved for the asymptotics. The second approach, based on the finite element method with a truncated perfectly matched layer, is not restricted to thin structures. We demonstrate the use of these methods in numerical calculations which further illustrate their differences. The asymptotic method finds resonance by solving a dense, but small, nonlinear eigenvalue problem, whereas the finite element method yields a large, but linear and sparse generalized eigenvalue problem. Both methods reproduce a localized defect mode found previously by finite difference time domain methods.
\end{abstract}

1. Introduction. This paper deals with the calculation of resonances of thin high contrast dielectric structures. Specifically, we are motivated by recent developments in photonic band gap (PBG) devices. PBG materials are artificially created structures having a refraction index which is spatially periodic, often on the nanoscale. As the name suggests, electromagnetic waves of frequencies in a 'band gap' cannot propagate within PBG materials. These materials thus offer interesting possibilities for radical manipulation of light through introduction of defects, hence the increasing interest in them.

While the existence of band gaps has been definitively demonstrated for certain infinite periodic structures, practical PBG devices are of finite extent. When a band gap exists in a medium of infinite extent, it is possible to create a so-called 'defect mode' which is a standing wave of frequency in the band gap by introducing a localized defect into the medium [6]. nSuch a mode corresponds to an eigenfunction of the partial differential equation governing the system. However, when the medium is of finite extent, such an eigenvalue no longer exists, but instead we may have a localized resonance mode.

A particularly interesting class of PBG structures are high index thin film devices where light is confined to the film by total internal reflection, and the PBG effect is achieved by drilling an array of air holes. Examples of such thin film devices can be found in $[5,19,20]$. The present work is aimed at calculating resonances for such structures. The dielectric properties of these structures are the restrictions of a periodic function to a bounded thin region in $\mathbb{R}^{3}$. Additionally they have a local "defect", i.e., a break in the periodic pattern. We want to identify resonance modes that are localized near the defect region, if any.

To model such structures, we consider the simplest equation for time harmonic

${ }^{*}$ This work was supported by the National Science Foundation under grants SCREMS-0619080, DMS-0749396, DMS-0713833, and DMS-0504185.

${ }^{\dagger}$ University of Florida, Department of Mathematics, Gainesville, FL 32611-8105, (jayg@math.ufl.edu).

${ }_{\ddagger}^{\ddagger}$ Department of Mathematics, Drexel University, Philadelphia, PA 19104-2875, (moskow@math.drexel.edu).

$\S$ School of Mathematics, University of Minnesota, Vincent Hall,Minneapolis, MN 55455, (santosa@math.umn.edu). 
wave propagation, namely the Helmholtz equation,

$$
\Delta u+k^{2} \varepsilon(\boldsymbol{x}) u=0, \quad \boldsymbol{x} \in \mathbb{R}^{n},
$$

where $n=2$ or 3 , and $u$ and $\varepsilon$ are functions of $\boldsymbol{x}$ in $\mathbb{R}^{n}$. By an abuse in terminology we will call $\varepsilon(\boldsymbol{x})$ the dielectric constant. The geometry is captured by the variable coefficient $\varepsilon(\boldsymbol{x})$ which is set to unity in the background (air). The function $\varepsilon(\boldsymbol{x})-1$ is assumed to have compact support. To find resonances, we must find a nontrivial 'radiating' mode $u$ and a complex number $\lambda \equiv k^{2}$ such that

$$
-\Delta u=\lambda \varepsilon(\boldsymbol{x}) u, \quad \text { in } \mathbb{R}^{n} .
$$

When $k$ is real, a mathematically precise form of the condition that $u$ is 'radiating' (or 'outgoing') is the well known Sommerfeld radiation condition at infinity. Writing (1.2) together with the Sommerfeld condition as $A u=\lambda B u$, the resolvent $(A-\lambda B)^{-1}$ is well defined for $\lambda$ in the positive real axis, because the Sommerfeld condition gives uniqueness of Helmholtz solutions. When $k($ or $\lambda$ ) is complex, one way to make the 'outgoing' condition precise is by analytic continuation from the positive real axis. For instance, for a slightly different scattering problem studied in [13], the resolvent was proved to be a meromorphic function of $\lambda$ and resonances were characterized as its poles occurring in the lower half of the complex plane.

The resonance modes $u$ satisfying equations like (1.2) are sometimes also known as quasi-normal modes [12]. They are non-physical and grow exponentially when $k$ is in the fourth quadrant. To give a physical interpretation of resonance, we must go to the time domain and consider

$$
\Delta U-\varepsilon(\boldsymbol{x}) U_{t t}=0, \quad \boldsymbol{x} \in \mathbb{R}^{n} .
$$

Resonance in this context is a time-dependent solution of the equation that resembles a standing wave except for the amplitude decay. Such a solution, especially when the decay is slow, is well captured by a superposition of quasi-normal modes with $e^{i k t}$ modulation [12]. Generally these slowly decaying resonance modes are computed by using finite difference time domain methods (FDTD), which are computationally intensive. In this work we propose two other ways to calculate resonances.

For thin devices, we propose a direct approach based on the Lippman-Schwinger reformulation. We assume a structure which fits into the following high contrast model, namely the dielectric occupies the region $\Omega \times(-h / 2, h / 2)$. In three dimensions, $\Omega$ is a bounded planar domain, while in two space dimensions, $\Omega$ is bounded domain on the real line. Thus, in either case, $\Omega$ is of one space dimension less than $n$. Let $\boldsymbol{x}=(x, z)$ for $x \in \mathbb{R}^{2}$ and $z \in \mathbb{R}$. We assume that

$$
\varepsilon(x, z)= \begin{cases}\frac{\varepsilon_{0}(x)}{h}, & \text { if }|z|<h / 2 \text { and } x \in \Omega \\ 1, & \text { otherwise. }\end{cases}
$$

Note that whenever we have a membrane whose dielectric properties vary negligibly across its thickness $h$, we can satisfy this assumption by setting $\varepsilon_{0}$ to $h \varepsilon$. In [14], we studied scattering by this type of structure, and found a limiting, or effective problem as $h \rightarrow 0$, with a correction term that improved the approximation. In this paper, we study the related resonance problem. 
We define the resonant frequency $k$ as a number in the complex plane for which there is a nontrivial resonance mode $u$ satisfying

$$
u(x, z)=\lambda \int_{\Omega} \int_{-h / 2}^{h / 2}\left(1-\frac{\varepsilon_{0}\left(x^{\prime}\right)}{h}\right) G_{\lambda}\left(x, z, x^{\prime}, z^{\prime}\right) u\left(x^{\prime}, z^{\prime}\right) d z^{\prime} d x^{\prime} .
$$

Here $\lambda \equiv k^{2}$ (which we will call the resonance value) and $G$ is the Helmholtz fundamental solution (in $2 \mathrm{D}$ or $3 \mathrm{D}$ ). One can show by variational arguments that such a $\lambda$ must necessarily be in the lower half plane, and hence $k$ must be in the fourth quadrant. This integral equation is arrived at from (1.1) by the same standard manipulations used in deriving the Lippman-Schwinger equation for scattering problems (see Theorem 8.3 of [4]). However, since $\lambda$ has negative imaginary part, the solutions to (1.4) are exponentially growing at infinity, and such manipulations are only formal. Nevertheless this suggests that the definition of resonances using (1.4) is equivalent to (1.2). As another way to see why this is the case [8], consider the operator

$$
\left(\Delta+k^{2} \epsilon\right)
$$

for real $k$. One can then write the outgoing Green's function to characterize the inverse of this operator with Sommerfeld radiation conditions,

$$
R(k)=\left(\Delta+k^{2} \epsilon\right)^{-1} .
$$

If one continues this operator to negative complex $k$, the classical definition of resonance is its poles. Now we can rewrite this operator as

$$
\begin{aligned}
R(k) & =\left(\Delta+k^{2}+k^{2}(\epsilon-1)\right)^{-1} \\
& =\left[\left(\Delta+k^{2}\right)\left(I+\left(\Delta+k^{2}\right)^{-1} k^{2}(\epsilon-1)\right)\right]^{-1} \\
& =\left[I+\left(\Delta+k^{2}\right)^{-1} k^{2}(\epsilon-1)\right]^{-1}\left(\Delta+k^{2}\right)^{-1} .
\end{aligned}
$$

Since the term $\left(\Delta+k^{2}\right)^{-1}$ is characterized by the free space Green's function, it has no poles. So, the poles of $R(k)$ are exactly where

$$
I+\left(\Delta+k^{2}\right)^{-1} k^{2}(\epsilon-1)
$$

has a null space, i.e. where (1.4) has a solution. Note that our theoretical analysis neither refers to nor deals with this equivalence. Indeed, our analysis takes (1.4) as the definition of resonances and proceeds to examine how such resonance values vary with $h$. Since $G$ depends on $\lambda,(1.4)$ is a nonlinear eigenvalue problem.

Another approach for numerical approximation of resonances is to directly approximate the eigenvalue problem in (1.1) with an outgoing boundary condition. A standard technique to handle outgoing boundary conditions at infinity is by introducing a perfectly matched layer [1] (PML) away from all inhomogeneities and eventually truncating the layer to obtain a finite computational domain. This suggests the use of PML for computing resonances by solving a linear eigenvalue problem in a truncated domain. We investigate this approach numerically, comparing the results with an exact solution as well as with approximations from the asymptotic approach. For the case of thin structures we can use both approaches to validate one another. A significant finding of this paper is that with both the asymptotic method and the PML calculations we can reproduce the high quality factor (low loss) resonance mode found in [5] by FDTD methods. 
The next section contains a derivation and analysis of an asymptotic approximation to resonance solutions of (1.4) with respect to the thickness parameter $h$. Within this section, we prove convergence of the related operators, convergence of the resonance values, and then finally derive a correction term for the resonances utilizing an eigenvalue approximation theorem of Osborn [16]. Section 3 contains a numerical study of both asymptotic/nonlinear eigenvalue and PML approaches to find the resonance solutions. In $\S 3.1$, we find exact solutions for the resonances of a disk and use them to analyze the convergence of PML solutions. In $\S 3.2$ we return to a thin high contrast structure. We compute resonances with both PML and asymptotics for the same problem and compare the results. In $\S 3.3$ we study a thin periodic structure with a defect from [5], which was previously found to exhibit a localized low loss mode. The concluding section summarizes our results.

2. An asymptotic limit. In this section we develop an asymptotic approach to the resonance approximation for these thin, high contrast structures. The resonance problem is then formulated in terms of operator equations, and we prove operator convergence, that is, we show that the operators depend continuously on the thickness and frequency parameters. In the subsections that follow, we show that the resonance values converge and prove an error estimate. In the case of a simple resonance value, we introduce a correction term that increases the accuracy of the asymptotic approximation.

Assume that we have a dielectric with geometry defined by (1.3). A resonance value $\lambda_{h}$ is a complex number for which there is a nontrivial function $u_{h}$ satisfying

$$
u_{h}(x, z)=\lambda_{h} \int_{\Omega} \int_{-h / 2}^{h / 2}\left(1-\frac{\varepsilon_{0}\left(x^{\prime}\right)}{h}\right) G_{\lambda_{h}}\left(x, z, x^{\prime}, z^{\prime}\right) u_{h}\left(x^{\prime}, z^{\prime}\right) d z^{\prime} d x^{\prime}
$$

where $\varepsilon_{0}$ is assumed to be piecewise continuous and $G$ is the Helmholtz fundamental solution (in 2 or $3 \mathrm{~d}$ ) with complex $\lambda_{h}=k^{2}$. That is, when $n=3$,

$$
G_{\lambda}\left(x, z, x^{\prime}, z^{\prime}\right)=-\frac{1}{4 \pi} \frac{e^{i \sqrt{\lambda} \sqrt{\left|x-x^{\prime}\right|^{2}+\left|z-z^{\prime}\right|^{2}}}}{\sqrt{\left|x-x^{\prime}\right|^{2}+\left|z-z^{\prime}\right|^{2}}}
$$

and when $n=2$,

$$
G_{\lambda}\left(x, z, x^{\prime}, z^{\prime}\right)=-\frac{i}{4} H_{0}^{(1)}\left(\sqrt{\lambda} \sqrt{\left|x-x^{\prime}\right|^{2}+\left|z-z^{\prime}\right|^{2}}\right)
$$

where $H_{0}^{(1)}$ is a Hankel function of the first kind. We note that we are taking the branch of the square root in the complex plane for which the cut is on the negative real axis, and hence there is analyticity away from this cut. With the scaling in the $z$ direction, $z=h \zeta$, let

$$
\tilde{u}_{h}(x, \zeta)=u_{h}(x, z)
$$

to obtain

$$
\tilde{u}_{h}(x, \zeta)=\lambda_{h} \int_{\Omega} \int_{-1 / 2}^{1 / 2}\left(h-\varepsilon_{0}\left(x^{\prime}\right)\right) G_{\lambda_{h}}\left(x, h \zeta, x^{\prime}, h \zeta^{\prime}\right) \tilde{u}_{h}\left(x^{\prime}, \zeta^{\prime}\right) d \zeta^{\prime} d x^{\prime} .
$$

Now, if we let $h \rightarrow 0$, this leads us to guess the limiting resonance problem: Find nontrivial solutions $\left(u_{0}, \lambda_{0}\right)$ to

$$
u_{0}(x)=-\lambda_{0} \int_{\Omega} \varepsilon_{0}\left(x^{\prime}\right) G_{\lambda_{0}}\left(x, 0, x^{\prime}, 0\right) u_{0}\left(x^{\prime}\right) d x^{\prime} .
$$


Although still a nonlinear eigenvalue problem, this has one dimension less than we started with.

In order to analyze the validity of this asymptotic limit, it is useful to express these problems in operator form. Let $S$ be the scaled, fixed domain

$$
S=\Omega \times[-1 / 2,1 / 2] .
$$

Consider, for $v \in L^{2}(S)$, the operators $T_{h}(\lambda)$ and $T_{0}(\lambda)$, with complex parameter $\lambda$, are defined by

$$
T_{h}(\lambda) v=\int_{-1 / 2}^{1 / 2} \int_{\Omega}\left(h-\varepsilon_{0}\left(x^{\prime}\right)\right) G_{\lambda}\left(x, h \zeta, x^{\prime}, h \zeta^{\prime}\right) v\left(x^{\prime}, \zeta^{\prime}\right) d x^{\prime} d \zeta^{\prime}
$$

and

$$
T_{0}(\lambda) v=-\int_{-1 / 2}^{1 / 2} \int_{\Omega} \varepsilon_{0}\left(x^{\prime}\right) G_{\lambda}\left(x, 0, x^{\prime}, 0\right) v\left(x^{\prime}, \zeta^{\prime}\right) d x^{\prime} d \zeta^{\prime} .
$$

The operators $T_{h}(\lambda)$ and $T_{0}(\lambda)$ are both compact from $L^{2}(S)$ to $L^{2}(S)$, by the proof of [14, Lemma 2]. (Unlike in that Lemma, here since $\lambda$ is not necessarily real, we are not ensured the invertibility of $\left(I-\lambda T_{h}(\lambda)\right)$ or $\left(I-\lambda T_{0}(\lambda)\right)$, hence the presence of resonance values.) We say that $\lambda_{h}$ is a resonance value of $T_{h}$ if there exists nontrivial $u_{h} \in L^{2}(S)$ such that

$$
u_{h}=\lambda_{h} T_{h}\left(\lambda_{h}\right) u_{h} .
$$

Similarly, $\lambda_{0}$ is a resonance value of $T_{0}$ if there exists nontrivial $u_{0}$ such that

$$
u_{0}=\lambda_{0} T_{0}\left(\lambda_{0}\right) u_{0}
$$

The operators $T_{h}, T_{0}$ are compact on $C^{0}(S)$ as well as $L^{2}(S)$, but here we will use $L^{2}(S)$ for its Hilbert space structure. We use $\langle$,$\rangle to denote the standard L^{2}(S)$ inner product over $\mathbb{C}$;

$$
\langle u, v\rangle:=\int_{S} u \bar{v}
$$

where $\bar{v}$ is the complex conjugate of $v$.

2.1. Operator convergence. We first prove a lemma showing convergence of the fundamental solutions when $n=3$. The same result also holds for $n=2$. This is an extension of [14, Lemma 1] to complex $\lambda$. Here we also give the explicit dependence of the constant on $\lambda$. Recall the definition of the scaled domain

$$
S=\Omega \times(-1 / 2,1 / 2)
$$

Lemma 2.1. There exists a constant $C$ independent of $h, \zeta^{\prime}$, and $\lambda$, such that

$$
\sup _{(x, \zeta) \in S} \int_{\Omega}\left|G_{\lambda}\left(x, 0, x^{\prime}, 0\right)-G_{\lambda}\left(x, h \zeta, x^{\prime}, h \zeta^{\prime}\right)\right| d x^{\prime} \leq C h(1+|\sqrt{\lambda}|) e^{|\mathcal{I} m \sqrt{\lambda}| \operatorname{diam}\left(\Omega_{h}\right)} .
$$


Proof The difference of these fundamental solutions can be written as

$$
\begin{aligned}
& G\left(x, h \zeta, x^{\prime}, h \zeta^{\prime}\right)-G\left(x, 0, x^{\prime}, 0\right) \\
& =\frac{1}{4 \pi} \frac{e^{i \sqrt{\lambda}\left|x-x^{\prime}\right|}}{\left|x-x^{\prime}\right|}-\frac{1}{4 \pi} \frac{e^{i \sqrt{\lambda} \sqrt{\left|x-x^{\prime}\right|^{2}+h^{2}\left|\zeta-\zeta^{\prime}\right|^{2}}}}{\sqrt{\left|x-x^{\prime}\right|^{2}+h^{2}\left|\zeta-\zeta^{\prime}\right|^{2}}} \\
& =\frac{1}{4 \pi} e^{i \sqrt{\lambda}\left|x-x^{\prime}\right|}\left[\frac{1}{\left|x-x^{\prime}\right|}-\frac{1}{\sqrt{\left|x-x^{\prime}\right|^{2}+h^{2}\left|\zeta-\zeta^{\prime}\right|^{2}}}\right] \\
& \quad+\frac{1}{4 \pi} \frac{1}{\sqrt{\left|x-x^{\prime}\right|^{2}+h^{2}\left|\zeta-\zeta^{\prime}\right|^{2}}}\left[e^{i \sqrt{\lambda}\left|x-x^{\prime}\right|}-e^{i \sqrt{\lambda} \sqrt{\left|x-x^{\prime}\right|^{2}+h^{2}\left|\zeta-\zeta^{\prime}\right|^{2}}}\right] .
\end{aligned}
$$

We first work on the second term on the right-hand side of (2.4). By a standard Taylor expansion,

$e^{i \sqrt{\lambda} \sqrt{\left|x-x^{\prime}\right|^{2}+h^{2}\left|\zeta-\zeta^{\prime}\right|^{2}}}=e^{i \sqrt{\lambda}\left|x-x^{\prime}\right|}+i \sqrt{\lambda}\left(\sqrt{\left|x-x^{\prime}\right|^{2}+h^{2}\left|\zeta-\zeta^{\prime}\right|^{2}}-\left|x-x^{\prime}\right|\right) e^{i \sqrt{\lambda} \xi}$

for some $\xi$ between $\left|x-x^{\prime}\right|$ and $\sqrt{\left|x-x^{\prime}\right|^{2}+h^{2}\left|\zeta-\zeta^{\prime}\right|^{2}}$. Since we know that for $(x, \zeta) \in S$,

$$
\sqrt{\left|x-x^{\prime}\right|^{2}+h^{2}\left|\zeta-\zeta^{\prime}\right|^{2}}-\left|x-x^{\prime}\right| \leq h
$$

we obtain

$$
\begin{aligned}
\mid e^{i \sqrt{\lambda}\left|x-x^{\prime}\right|}-e^{i \sqrt{\lambda} \sqrt{\left|x-x^{\prime}\right|^{2}+h^{2}\left|\zeta-\zeta^{\prime}\right|^{2}} \mid} & \leq|\sqrt{\lambda}| h e^{|\mathcal{I} m \sqrt{\lambda}| \xi} \\
& \leq|\sqrt{\lambda}| h e^{|\mathcal{I} m \sqrt{\lambda}| \operatorname{diam}\left(\Omega_{h}\right)} .
\end{aligned}
$$

Also,

$$
\frac{1}{\sqrt{\left|x-x^{\prime}\right|^{2}+h^{2}\left|\zeta-\zeta^{\prime}\right|^{2}}} \leq \frac{1}{\left|x-x^{\prime}\right|}
$$

which is integrable with respect to $x^{\prime}$ on $\Omega$, we have that

$$
\int_{\Omega} \frac{d x^{\prime}}{\sqrt{\left|x-x^{\prime}\right|^{2}+h^{2}\left|\zeta-\zeta^{\prime}\right|^{2}}}
$$

is bounded independently of $h, \zeta^{\prime}, \lambda$, and $(x, z) \in S$. This along with (2.5) gives that we can choose $C$ independent of $h, \zeta^{\prime}$ and $(x, \zeta) \in S$ such that

$$
\int_{\Omega} \frac{1}{4 \pi} \frac{\mid e^{i \sqrt{\lambda}\left|x-x^{\prime}\right|}-e^{i \sqrt{\lambda} \sqrt{\left|x-x^{\prime}\right|^{2}+h^{2}\left|\zeta-\zeta^{\prime}\right|^{2}} \mid}}{\sqrt{\left|x-x^{\prime}\right|^{2}+h^{2}\left|\zeta-\zeta^{\prime}\right|^{2}}} d x^{\prime} \leq C h|\sqrt{\lambda}| e^{|\mathcal{J} m \sqrt{\lambda}| \operatorname{diam}\left(\Omega_{h}\right)}
$$

The integral of the first term on the right-hand side of (2.4) can be bounded

$$
\begin{aligned}
& \int_{\Omega}\left|\frac{1}{4 \pi} e^{i \sqrt{\lambda}\left|x-x^{\prime}\right|}\left[\frac{1}{\left|x-x^{\prime}\right|}-\frac{1}{\sqrt{\left|x-x^{\prime}\right|^{2}+h^{2}\left|\zeta-\zeta^{\prime}\right|^{2}}}\right]\right| d x^{\prime} \\
& \leq \frac{1}{4 \pi} e^{|\mathcal{I} m \sqrt{\lambda}| \operatorname{diam}\left(\Omega_{h}\right)} \int_{\Omega} \mid \frac{1}{\left|x-x^{\prime}\right|}-\frac{1}{\sqrt{\left|x-x^{\prime}\right|^{2}+h^{2}\left|\zeta-\zeta^{\prime}\right|^{2}} \mid} d x^{\prime} \\
& =\frac{1}{4 \pi} e^{|\mathcal{J} m \sqrt{\lambda}| \operatorname{diam}\left(\Omega_{h}\right)} \int_{\Omega}\left(\frac{1}{\left|x-x^{\prime}\right|}-\frac{1}{\sqrt{\left|x-x^{\prime}\right|^{2}+h^{2}\left|\zeta-\zeta^{\prime}\right|^{2}}}\right) d x^{\prime}
\end{aligned}
$$


since the integrand is non-negative. Now choose $R$ large enough so that if $B_{R}(x)$ is the ball of radius $R$ centered at $x$ in $\mathbb{R}^{2}$,

$$
\Omega \subset B_{R}(x)
$$

for all $x \in \Omega$. Then the integral over $\Omega$ above is bounded by

$$
\leq \int_{B_{R}(x)}\left(\frac{1}{\left|x-x^{\prime}\right|}-\frac{1}{\sqrt{\left|x-x^{\prime}\right|^{2}+h^{2}\left|\zeta-\zeta^{\prime}\right|^{2}}}\right) d x^{\prime} .
$$

Change to polar coordinates centered at $x$ with

$$
r=\left|x-x^{\prime}\right| .
$$

The integral transforms to

$$
\begin{aligned}
& =2 \pi \int_{0}^{R}\left(\frac{1}{r}-\frac{1}{\sqrt{r^{2}+h^{2}\left|\zeta-\zeta^{\prime}\right|^{2}}}\right) \\
& =2 \pi\left[R-\sqrt{R^{2}+h^{2}\left|\zeta-\zeta^{\prime}\right|^{2}}+h\left|\zeta-\zeta^{\prime}\right|\right]
\end{aligned}
$$

by direct calculation. One can see clearly that this quantity is then $O(h)$, where the constant is independent of $(x, \zeta) \in S, \lambda$, and $\zeta^{\prime} \in(-1 / 2,1 / 2)$. This, combined with (2.4) and the estimate (2.6) proves the lemma.

Next we show that the operators depend continuously on the parameters $h$ and $\lambda$.

Proposition 2.1. Assume we have a sequence of pairs $\left\{h_{j}, \lambda_{j}\right\}$, where $h_{j} \in \mathbb{R}$, the $\lambda_{j}$ are in the complex plane with the negative real axis and the origin removed, i.e. $\lambda_{j} \in \mathbb{C} \backslash\left\{\mathbb{R}^{-} \cup\{0\}\right\}$, and for which $\lambda_{j} \rightarrow \lambda_{0}$ for some $\lambda_{0} \in \mathbb{C} \backslash\left\{\mathbb{R}^{-} \cup\{0\}\right\}$, and $h_{j} \rightarrow 0$ as $j \rightarrow \infty$. Then

$$
T_{h_{j}}\left(\lambda_{j}\right) \rightarrow T_{0}\left(\lambda_{0}\right)
$$

in the operator norm on $L^{2}(S)$ as $j \rightarrow \infty$. Furthermore, for $j$ large enough there exists $C$ independent of $j$ such that

$$
\left\|T_{h_{j}}\left(\lambda_{j}\right)-T_{0}\left(\lambda_{0}\right)\right\| \leq C\left(h_{j}+\left|\lambda_{j}-\lambda_{0}\right|\right) .
$$

Proof Consider, for $v \in L^{2}(S)$,

$$
\left(T_{h_{j}}\left(\lambda_{j}\right)-T_{0}\left(\lambda_{0}\right)\right) v=\left(T_{h_{j}}\left(\lambda_{j}\right)-T_{0}\left(\lambda_{j}\right)\right) v+\left(T_{0}\left(\lambda_{j}\right)-T_{0}\left(\lambda_{0}\right)\right) v .
$$

We will expand out the second term on the right hand side

$$
\left(T_{0}\left(\lambda_{j}\right)-T_{0}\left(\lambda_{0}\right)\right) v=\int_{-1 / 2}^{1 / 2} \int_{\Omega} \varepsilon_{0}\left(x^{\prime}\right)\left(G_{\lambda_{0}}\left(x, 0, x^{\prime}, 0\right)-G_{\lambda_{j}}\left(x, 0, x^{\prime}, 0\right)\right) v\left(x^{\prime}, \zeta^{\prime}\right) d x^{\prime} d \zeta^{\prime} .
$$

We can use the Mean Value Theorem, for $\left|x-x^{\prime}\right| \neq 0$,

$$
\begin{aligned}
G_{\lambda_{j}}\left(x, 0, x^{\prime}, 0\right)-G_{\lambda_{0}}\left(x, 0, x^{\prime}, 0\right) & =\frac{1}{4 \pi} \frac{e^{i \sqrt{\lambda_{0}}\left|x-x^{\prime}\right|}}{\left|x-x^{\prime}\right|}-\frac{1}{4 \pi} \frac{e^{i \sqrt{\lambda_{j}}\left|x-x^{\prime}\right|}}{\left|x-x^{\prime}\right|} \\
& =\left(\lambda_{0}-\lambda_{j}\right) \frac{i}{8 \pi \sqrt{\eta}} e^{i \sqrt{\eta}\left|x-x^{\prime}\right|}
\end{aligned}
$$


for some $\eta$ on the line in $\mathbb{C}$ joining $\lambda_{0}$ and $\lambda_{j}$. So, since $\lambda_{0}$ is bounded away from the negative real axis, for large enough $j$ we have

$$
\left\|G_{\lambda_{0}}\left(x, 0, x^{\prime}, 0\right)-G_{\lambda_{j}}\left(x, 0, x^{\prime}, 0\right)\right\|_{\infty} \leq C\left|\lambda_{0}-\lambda_{j}\right|
$$

for some $C$ independent of $j$. From this we easily obtain

$$
\left\|\left(T_{0}\left(\lambda_{j}\right)-T_{0}\left(\lambda_{0}\right)\right) v\right\|_{L^{2}(S)} \leq C\left|\lambda_{0}-\lambda_{j}\right|\|v\|_{L^{2}(S)} .
$$

Now, for the other term,

$$
\begin{aligned}
\left(T_{h_{j}}\left(\lambda_{j}\right)-\right. & \left.T_{0}\left(\lambda_{j}\right)\right) v=h_{j} \int_{S} G_{\lambda_{j}}\left(x, h_{j} \zeta, x^{\prime}, h_{j} \zeta^{\prime}\right) v\left(x^{\prime}, \zeta^{\prime}\right) d x^{\prime} d \zeta^{\prime} \\
& \quad-\int_{S} \varepsilon_{0}\left(x^{\prime}\right)\left[G_{\lambda_{j}}\left(x, h_{j} \zeta, x^{\prime}, h_{j} \zeta^{\prime}\right)-G_{\lambda_{j}}\left(x, 0, x^{\prime}, 0\right)\right] v\left(x^{\prime} \zeta^{\prime}\right) d x^{\prime} d \zeta^{\prime}
\end{aligned}
$$

Now, since $G_{\lambda_{j}}$ is a kernel which is bounded in $L^{1}$ independently of $h$, it follows from Generalized Young's Inequality [7] that the function

$$
w(x, \zeta)=\int_{S} G_{\lambda_{j}}\left(x, h_{j} \zeta, x^{\prime}, h_{j} \zeta^{\prime}\right) v\left(x^{\prime}, \zeta^{\prime}\right) d x^{\prime} d \zeta^{\prime}
$$

satisfies

$$
\|w\|_{L^{2}(S)} \leq C\|v\|_{L^{2}(S)},
$$

which shows that the first term in $(2.8)$ is $O\left(h_{j}\right)$. For the second term, we appeal to Lemma 2.1, which tells us that the kernel difference can be bounded:

$$
\left\|G_{\lambda_{j}}\left(x, h_{j} \zeta, x^{\prime}, h_{j} \zeta^{\prime}\right)-G_{\lambda_{j}}\left(x, 0, x^{\prime}, 0\right)\right\|_{L^{1}(S)} \leq C h_{j}
$$

where $C$ is independent of $j$. Since $\varepsilon_{0}$ is bounded in $L^{\infty}$, again using Generalized Young and the triangle inequalities in (2.8) we obtain,

$$
\left\|\left(T_{h_{j}}\left(\lambda_{j}\right)-T_{0}\left(\lambda_{j}\right)\right) v\right\|_{L^{2}(S)} \leq C h_{j}\|v\|_{L^{2}(S)} .
$$

Combining this with (2.7), the result follows.

2.2. Convergence of the resonance values. Define the modified resolvent type operator valued functions on $\mathbb{C}$

$$
R_{h}(\lambda)=\left(I-\lambda T_{h}(\lambda)\right)^{-1}
$$

and

$$
R_{0}(\lambda)=\left(I-\lambda T_{0}(\lambda)\right)^{-1}
$$

An important note is that if $R_{h}(\lambda)$ does not exist as a bounded linear operator from $L^{2}(S)$ to itself, then $\lambda$ is a resonance value of $T_{h}$. This is because if $R_{h}(\lambda)$ does not exist, then $1 / \lambda$ is in the spectrum of the compact operator $T_{h}(\lambda)$. Hence $1 / \lambda$ must be an eigenvalue, and $\left(I-\lambda T_{h}(\lambda)\right)$ must have nontrivial and finite dimensional null space. The same holds for the limiting operator $R_{0}(\lambda)$.

In the following theorem, we show that, with an assumption of nonzero residue, the resonance values converge. 
TheOREm 2.2. Assume that $\lambda_{0}$ is a resonance value of $T_{0}$, and that $R_{0}$ and $R_{h}$ are meromorphic in some region of $\mathbb{C}$ containing $\lambda_{0}$. Assume also that $R_{0}$ has nonzero residue at $\lambda_{0}$. Then for any ball $B$ around $\lambda_{0}$, there exists $h_{0}>0$ such that $T_{h}$ has a resonance in $B$ for all $h<h_{0}$. Conversely, if $\left\{\lambda_{h}\right\}$ is a sequence of resonance values of $T_{h}$ that converges as $h \rightarrow 0$, the limit is a resonance value of $T_{0}$. Proof We first note that we know from [14] that $\lambda_{0} \notin \mathbb{R}$. So, we can choose $B$, a ball around $\lambda_{0}$ which does not intersect the negative real axis $\mathbb{R}^{-}$, and such that $T_{0}$ has no other resonance values in $\bar{B}$.

We will also use a well known result about the inverses of perturbed operators, see for example [10] p. 31: If $S-T=A$ and $T^{-1}$ exists, then for $\|A\|<\frac{1}{\| T^{-1}}, S^{-1}$ exists and

$$
\left\|S^{-1}-T^{-1}\right\| \leq \frac{\|A\|\left\|T^{-1}\right\|^{2}}{1-\|A\|\left\|T^{-1}\right\|}
$$

Apply this, with

$$
\begin{aligned}
& S=I-\lambda T_{h}(\lambda) \\
& T=I-\lambda T_{0}(\lambda)
\end{aligned}
$$

to get

$$
\left\|R_{h}(\lambda)-R_{0}(\lambda)\right\| \leq \frac{\left\|\lambda\left(T_{0}(\lambda)-T_{h}(\lambda)\right)\right\|\left\|R_{0}(\lambda)\right\|^{2}}{1-\left\|\lambda\left(T_{0}(\lambda)-T_{h}(\lambda)\right)\right\|\left\|R_{0}(\lambda)\right\|}
$$

which, from Proposition 2.11 yields

$$
\left\|R_{h}(\lambda)-R_{0}(\lambda)\right\| \leq \frac{C h\left\|R_{0}(\lambda)\right\|^{2}}{1-C h\left\|R_{0}(\lambda)\right\|}
$$

for $C$ independent of $h$, for $h$ small enough. The constant $C$ does in general depend on $\lambda$, but for $\lambda$ on a compact subset of $\mathbb{C}$ bounded away from the real line, $C$ can be chosen independent of $\lambda$.

Let $\Gamma=\partial B$, positively oriented. By the choice of $B, \Gamma$ does not intersect with $\mathbb{R}^{-}$, and $\lambda_{0}$ is the only pole of $R_{0}$ in the closed disk. Then $R_{0}(\lambda)$ is continuous with respect to $\lambda$ on $\Gamma$, hence $\left\|R_{0}(\lambda)\right\|$ is uniformly bounded for $\lambda$ on $\Gamma$. Using (2.10), we have that

$$
R_{h}(\lambda) \rightarrow R_{0}(\lambda)
$$

in norm as $h \rightarrow 0$, uniformly for $\lambda \in \Gamma$. This implies that the operator valued integral

$$
\frac{1}{2 \pi i} \int_{\Gamma} R_{h}(\lambda) d \lambda \rightarrow \frac{1}{2 \pi i} \int_{\Gamma} R_{0}(\lambda) d \lambda
$$

in norm as $h \rightarrow 0$. From the residue theorem, the integral

$$
\frac{1}{2 \pi i} \int_{\Gamma} R_{0}(\lambda) d \lambda
$$

gives us the coefficient of the $\left(\lambda-\lambda_{0}\right)^{-1}$ term in the Laurent series expansion for $R_{0}(\lambda)$, which by assumption is nonzero. Hence the integrals must all be nonzero for 
$h$ small enough. This means that all $R_{h}$ must have at least one pole in $B$ for $h$ small enough. That is, for $h$ small enough, all $T_{h}$ have a resonance value in $B$. This proves the first part of the statement of the proposition. For the converse, if $\lambda_{0}$ is not a resonance value of $T_{0}$, then $R_{0}(\lambda)$ exists in some neighborhood of $\lambda_{0}$. The formula (2.10) implies that $R_{h}(\lambda)$ also exists in that neighborhood for $h$ small enough. Hence the resonance values of $T_{h}$ are bounded away from $\lambda_{0}$ for $h$ small enough.

Some remarks about the assumptions in this theorem:

- The operator functions $I-\lambda T_{h}(\lambda)$ and $I-\lambda T_{0}(\lambda)$ are analytic with respect to $\lambda$ away from the negative real axis. This, combined with the fact that the $T$ 's are compact, means that the inverses are meromorphic.

- If $\lambda_{0}$ is a resonance of $T_{0}$, then the classical resolvent of $T_{0}\left(\lambda_{0}\right)$, given by $\left(z I-T_{0}\left(\lambda_{0}\right)\right)^{-1}$, automatically has nonzero residue at $z=\frac{1}{\lambda_{0}}$; its residue is the projection onto the generalized eigenspace [10]. It is not clear how the residue of $R_{0}$ relates to the nonlinear eigenspace. However, if $\lambda_{0}$ is a simple pole, this is a special case of nonzero residue.

- We do not need the assumption about nonzero residue for the converse.

2.3. A higher order correction. Once we know that we have a convergent sequence of resonance values as $h \rightarrow 0$, we can use standard eigenvalue perturbation theorems. In the resonance value expansion, we employ a result of Osborn [16] which is valid for non-selfadjoint operators and also yields a correction term. The actual result in [16] is more general, but we state it here for the case of norm convergence on a Hilbert space.

Suppose $X$ is a Hilbert space and $T_{n}: X \rightarrow X$ is a sequence of compact linear operators such that $T_{n} \rightarrow T$ in norm. It then follows that the adjoint operators also converge in norm. Let $\mu$ be a nonzero eigenvalue of $T$ of algebraic multiplicity $m$. It is well known that for $n$ large enough, there exist $m$ eigenvalues of $T_{n}, \mu_{1}^{n}, \ldots \mu_{m}^{n}$ (counted according to algebrai multiplicity) such that $\mu_{j}^{n} \rightarrow \mu$ as $n \rightarrow \infty$, for each $1 \leq j \leq m$.

Let $E$ be the spectral projection onto the generalized eigenspace of $T$ corresponding to eigenvalue $\mu$. The space $X$ can be decomposed in terms of the range and null space of $E: X=R(E) \oplus N(E)$.

TheOREM 2.3 (Osborn). Let $\phi_{1}, \phi_{2}, \ldots \phi_{m}$ be a normalized basis for $R(E)$. Then there exists a constant $C$ such that

$$
\left|\mu-\frac{1}{m} \sum_{j=1}^{m} \mu_{j}^{n}-\frac{1}{m} \sum_{j=1}^{m}\left\langle\left(T-T_{n}\right) \phi_{j}, \phi_{j}\right\rangle\right| \leq C\left\|\left.\left(T-T_{n}\right)\right|_{R(E)}\right\| \cdot\left\|\left.\left(T^{*}-T_{n}^{*}\right)\right|_{R\left(E^{*}\right)}\right\|
$$

To simplify the statement of the following theorem, we define the lower dimensional operator $D T_{0}(\lambda)$ by

$$
D T_{0}(\lambda) v=-\int_{\Omega} \varepsilon_{0}\left(x^{\prime}\right) \frac{\partial G}{\partial \lambda}\left(x, 0, x^{\prime}, 0\right) v\left(x^{\prime}\right) d x^{\prime},
$$

and we leave off the subscripts $j$ for the sequence $\left\{h_{j}\right\}$ of values of $h$ going to zero.

TheOrem 2.4. Assume we have a sequence $\left\{\lambda_{h}\right\} \in \mathbb{C}$ of resonance values of $T_{h}$ for which $\lambda_{h} \rightarrow \lambda_{0}$ as $h \rightarrow 0$, where $\lambda_{0} \in \mathbb{C}$ is a simple resonance value of $T_{0}$ with normalized resonance function $u_{0}$ satisfying $\lambda_{0} T_{0}\left(\lambda_{0}\right) u_{0}=u_{0}$. Assume also that

$$
\lambda_{0}^{2}\left\langle D T_{0}\left(\lambda_{0}\right) u_{0}, u_{0}\right\rangle \neq-1 .
$$


Then there exists $C$ independent of $h$ such that

$$
\left|\lambda_{h}-\lambda_{0}\right| \leq C h
$$

and furthermore

$$
\lambda_{h}=\lambda_{0}+\lambda_{0}^{2} \frac{\left\langle\left(T_{0}\left(\lambda_{0}\right)-T_{h}\left(\lambda_{0}\right)\right) u_{0}, u_{0}\right\rangle}{1+\lambda_{0}^{2}\left\langle D T_{0}\left(\lambda_{0}\right) u_{0}, u_{0}\right\rangle}+O\left(h^{2}\right)
$$

Proof Note that

$$
\lambda_{h} T_{h}\left(\lambda_{h}\right) u_{h}=u_{h}
$$

and

$$
\lambda_{0} T_{0}\left(\lambda_{0}\right) u_{0}=u_{0},
$$

that is, $\frac{1}{\lambda_{h}}$ is an eigenvalue of $T_{h}\left(\lambda_{h}\right)$ and $\frac{1}{\lambda_{0}}$ is an eigenvalue of $T_{0}\left(\lambda_{0}\right)$. Also, from Proposition 2.1, we know that

$$
T_{h}\left(\lambda_{h}\right) \rightarrow T_{0}\left(\lambda_{0}\right)
$$

in the operator norm. So, what we have are the eigenvalues of a convergent sequence of compact operators. These operators, $\left\{T_{h}\left(\lambda_{h}\right), T_{0}\left(\lambda_{0}\right)\right\}$, are not self-adjoint, but it follows from the norm convergence that the adjoints also converge:

$$
T_{h}^{*}\left(\lambda_{h}\right) \rightarrow T_{0}^{*}\left(\lambda_{0}\right)
$$

in the operator norm, with the same norm error. Since we assume that $\frac{1}{\lambda_{0}}$ is a simple eigenvalue of $T_{0}\left(\lambda_{0}\right)$, Theorem 2.3 yields

$$
\begin{aligned}
\mid \frac{1}{\lambda_{0}}-\frac{1}{\lambda_{h}}- & \left\langle\left(T_{0}\left(\lambda_{0}\right)-T_{h}\left(\lambda_{h}\right)\right) u_{0}, u_{0}\right\rangle \mid \\
& \leq\left\|\left(T_{0}\left(\lambda_{0}\right)-T_{h}\left(\lambda_{h}\right)\right) u_{0}\right\| \cdot\left\|\left(T_{0}^{*}\left(\lambda_{0}\right)-T_{h}^{*}\left(\lambda_{h}\right)\right) u_{0}\right\|,
\end{aligned}
$$

Since

$$
\left\|T_{0}^{*}\left(\lambda_{0}\right)-T_{h}^{*}\left(\lambda_{h}\right)\right\|=\left\|T_{0}\left(\lambda_{0}\right)-T_{h}\left(\lambda_{h}\right)\right\|,
$$

we have from Proposition 2.1,

$$
\left|\frac{1}{\lambda_{0}}-\frac{1}{\lambda_{h}}-\left\langle\left(T_{0}\left(\lambda_{0}\right)-T_{h}\left(\lambda_{h}\right)\right) u_{0}, u_{0}\right\rangle\right| \leq C\left(h+\left|\lambda_{0}-\lambda_{h}\right|\right)^{2} .
$$

If we multiply everything by $\lambda_{0} \lambda_{h}$,

$$
\left|\lambda_{h}-\lambda_{0}-\lambda_{0} \lambda_{h}\left\langle\left(T_{0}\left(\lambda_{0}\right)-T_{h}\left(\lambda_{h}\right)\right) u_{0}, u_{0}\right\rangle\right| \leq C\left(h+\left|\lambda_{0}-\lambda_{h}\right|\right)^{2},
$$

which we manipulate to get

$$
\begin{aligned}
\lambda_{h}=\lambda_{0}+\lambda_{0}^{2}\left\langle\left(T_{0}\left(\lambda_{0}\right)-T_{h}\left(\lambda_{h}\right)\right) u_{0}, u_{0}\right\rangle & \\
& +\lambda_{0}\left(\lambda_{h}-\lambda_{0}\right)\left\langle\left(T_{0}\left(\lambda_{0}\right)-T_{h}\left(\lambda_{h}\right)\right) u_{0}, u_{0}\right\rangle+O\left(\left(h+\left|\lambda_{h}-\lambda_{0}\right|\right)^{2}\right) .
\end{aligned}
$$


Again using Proposition 2.1,

$$
\lambda_{h}=\lambda_{0}+\lambda_{0}^{2}\left\langle\left(T_{0}\left(\lambda_{0}\right)-T_{h}\left(\lambda_{h}\right)\right) u_{0}, u_{0}\right\rangle+O\left(\left(h+\left|\lambda_{h}-\lambda_{0}\right|\right)^{2}\right) .
$$

Now, since the correction term above depends on $\lambda_{h}$, we need to expand the term further. We can write

$$
T_{0}\left(\lambda_{0}\right)-T_{h}\left(\lambda_{h}\right)=\left(T_{0}\left(\lambda_{0}\right)-T_{h}\left(\lambda_{0}\right)\right)+\left(T_{h}\left(\lambda_{0}\right)-T_{h}\left(\lambda_{h}\right)\right)
$$

and compute

$$
\left(T_{h}\left(\lambda_{0}\right)-T_{h}\left(\lambda_{h}\right)\right)=\int_{S}\left(h-\varepsilon_{0}\left(x^{\prime}\right)\right)\left(G_{\lambda_{0}}-G_{\lambda_{h}}\right)\left(x, h \zeta, x^{\prime}, h \zeta^{\prime}\right) u_{0}\left(x^{\prime}\right) d \zeta^{\prime} d x^{\prime} .
$$

Note that since the range of $T_{0}$ contains only functions that are independent of $\zeta, u_{0}$ must be independent of $\zeta$. Since we are bounded away from the negative real axis, $G$ is analytic with respect to $\lambda$ and so by standard Taylor expansion we obtain

$$
\left(T_{h}\left(\lambda_{0}\right)-T_{h}\left(\lambda_{h}\right)\right)=\left.\int_{S}\left(h-\varepsilon_{0}\left(x^{\prime}\right)\right)\left(\lambda_{0}-\lambda_{h}\right) \frac{\partial G}{\partial \lambda}\right|_{\lambda=\lambda_{0}}\left(x, h \zeta, x^{\prime}, h \zeta^{\prime}\right) u_{0}\left(x^{\prime}\right) d x^{\prime} d \zeta^{\prime}+O\left(\left|\lambda_{0}-\lambda_{h}\right|^{2}\right) .
$$

Note that the integrand is now continuous. This yields, after expanding the exponential or Hankel function about $h=0$,

$$
\left(T_{h}\left(\lambda_{0}\right)-T_{h}\left(\lambda_{h}\right)\right)=\left.\int_{S} \varepsilon_{0}\left(x^{\prime}\right)\left(\lambda_{h}-\lambda_{0}\right) \frac{\partial G}{\partial \lambda}\right|_{\lambda=\lambda_{0}}\left(x, 0, x^{\prime}, 0\right) u_{0}\left(x^{\prime}\right) d x^{\prime} d \zeta^{\prime}+O\left(\left(h+\left|\lambda_{0}-\lambda_{h}\right|\right)^{2}\right) .
$$

Note the above integrand is independent of $\zeta^{\prime}$. Combining (2.17), (2.16), and (2.15),

$\lambda_{h}=\lambda_{0}+\lambda_{0}^{2}\left\langle\left(T_{0}\left(\lambda_{0}\right)-T_{h}\left(\lambda_{0}\right)\right) u_{0}, u_{0}\right\rangle-\lambda_{0}^{2}\left(\lambda_{h}-\lambda_{0}\right)\left\langle D T_{0}\left(\lambda_{0}\right) u_{0}, u_{0}\right\rangle+O\left(\left(h+\left|\lambda_{0}-\lambda_{h}\right|\right)^{2}\right)$

where $D T_{0}$ is defined by $(2.11)$. We now collect terms for $\left(\lambda_{h}-\lambda_{0}\right)$ to get

$\left(\lambda_{h}-\lambda_{0}\right)\left(1+\lambda_{0}^{2}\left\langle D T_{0}\left(\lambda_{0}\right) u_{0}, u_{0}\right\rangle\right)=\lambda_{0}^{2}\left\langle\left(T_{0}\left(\lambda_{0}\right)-T_{h}\left(\lambda_{0}\right)\right) u_{0}, u_{0}\right\rangle+O\left(\left(h+\left|\lambda_{0}-\lambda_{h}\right|\right)^{2}\right)$.

At this point we need to use the assumption (2.12) to obtain

$$
\lambda_{h}=\lambda_{0}+\frac{\lambda_{0}^{2}\left\langle\left(T_{0}\left(\lambda_{0}\right)-T_{h}\left(\lambda_{0}\right)\right) u_{0}, u_{0}\right\rangle}{1+\lambda_{0}^{2}\left\langle D T_{0}\left(\lambda_{0}\right) u_{0}, u_{0}\right\rangle}+O\left(\left(h+\left|\lambda_{0}-\lambda_{h}\right|\right)^{2}\right) .
$$

Recall that by the proof of Proposition 2.1,

$$
\left\|T_{0}\left(\lambda_{0}\right)-T_{h}\left(\lambda_{0}\right)\right\|=O(h)
$$

in the operator norm, and so we have

$$
\lambda_{h}-\lambda_{0}=O(h)+O\left(\left(h+\left|\lambda_{0}-\lambda_{h}\right|\right)^{2}\right) .
$$

Since we assume that $\lambda_{h}-\lambda_{0} \rightarrow 0$, this can only hold if

$$
\lambda_{h}-\lambda_{0}=O(h) \text {. }
$$

This completes the proof.

REMARK 2.1. It is possible that the hypothesis (2.12) is related to the residue of the generalized resolvent $R_{0}(\lambda)$. In particular, we conjecture that for a simple 
resonance value, $(2.12)$ holds if and only if the residue is nonzero, i.e. the requirement for convergence in Proposition 2.2.

The correction term above is not difficult to compute since it involves only applying integral operators to the limiting resonance function $u_{0}$. However, one may want to have an expression of the form

$$
\lambda_{h} \approx \lambda_{0}+h \lambda^{(1)}
$$

in which $\lambda^{(1)}$ is independent of $h$. The numerator of the correction we have studied exactly in [14], for the case when $\lambda_{0}$ was real. By that same analysis,

$$
\begin{gathered}
\left(T_{0}\left(\lambda_{0}\right)-T_{h}\left(\lambda_{0}\right)\right) u_{0}=-h \int_{\Omega} \int_{-1 / 2}^{1 / 2} G_{\lambda_{0}}\left(x, h \zeta, x^{\prime}, h \zeta^{\prime}\right) u_{0} d \zeta^{\prime} d x^{\prime} \\
+\int_{\Omega} \int_{-1 / 2}^{1 / 2} \varepsilon_{0}\left(x^{\prime}\right)\left(G_{\lambda_{0}}\left(x, h \zeta, x^{\prime}, h \zeta^{\prime}\right)-G_{\lambda_{0}}\left(x, 0, x^{\prime}, 0\right)\right) u_{0}\left(x^{\prime}\right) d \zeta^{\prime} d x^{\prime} \\
=-h \int_{\Omega} G_{\lambda_{0}}\left(x, 0, x^{\prime}, 0\right) u_{0}\left(x^{\prime}\right) d x^{\prime} \\
+h \frac{\varepsilon_{0}(x) u_{0}(x)}{2}\left(\zeta^{2}+\frac{1}{4}\right)+o(h) .
\end{gathered}
$$

This yields the following corollary.

Corollary 2.5. Assume the hypotheses as in Theorem 2.4. Then

$$
\lambda_{h}=\lambda_{0}+h \lambda_{0}^{2} \frac{\left\langle g, u_{0}\right\rangle}{1+\lambda_{0}^{2}\left\langle D T_{0}\left(\lambda_{0}\right) u_{0}, u_{0}\right\rangle}+o(h),
$$

where

$$
g(x)=-\int_{\Omega} G_{\lambda_{0}}\left(x, 0, x^{\prime}, 0\right) u_{0}\left(x^{\prime}\right) d x^{\prime}+\frac{\varepsilon_{0}(x) u_{0}(x)}{2}\left(\zeta^{2}+\frac{1}{4}\right) .
$$

Note however that here the error is no longer guaranteed to be $O\left(h^{2}\right)$.

3. Numerical techniques. In this section, we investigate two numerical approaches for the computation of resonances of thin membranes. The first is via Bérenger's perfectly matched layer (PML) [1]. The second is a collocation discretization of the integral equation formulation combined with the asymptotics developed in the previous section. The numerical analyses of both these approaches for resonance computation are presently open. Nonetheless, considerable insight into these computational approaches can be gained by comparing them with each other.

First, we will exhibit an example with a disk where we can compute resonances exactly. We will compute the approximations to these exact resonances using the PML approach and compare. Since no error analysis is known for the PML eigenvalue approximations, this will serve as validation of our first approach. Note that although PML has been increasingly used for computation of open resonances [9, 11, 17], we have not been able to locate a comparison of approximate and exact resonances in the literature - another reason for including such a comparison here.

Next, we will examine thin high contrast homogeneous structures. Here will investigate the asymptotic integral equation approach along with PML. While the PML 
approach reduces to a large sparse generalized eigenvalue computation, the second approach yields a small dense nonlinear eigenvalue problem. We will establish that the asymptotics are sound by testing the results against the ones obtained from PML.

Finally, we will use both the PML approach and asymptotics to compute a resonance mode found in [5] for a periodic structure with a defect. The mode is localized near the defect, that is, it exhibits photonic band gap type behavior. Also, it has a high quality factor, indicating that in the time domain its decay is slow. In [5] the mode was computed using a FDTD (finite difference time domain) method, as is typically the case.

3.1. A disk: exact resonances and PML validation.. We will now calculate the first few resonance modes of a circular homogeneous dielectric disk of radius $a$ having (constant) permittivity $\varepsilon_{d}$ placed in an infinite vacuum. If the mode is written in the form

$$
U(\boldsymbol{x}, t)=e^{-i k t} u(\boldsymbol{x})= \begin{cases}e^{-i k t} u^{+}(\boldsymbol{x}), & |\boldsymbol{x}|>a, \\ e^{-i k t} u^{-}(\boldsymbol{x}), & |\boldsymbol{x}| \leq a,\end{cases}
$$

the governing equations are

$$
\begin{aligned}
\Delta u^{+}+k^{2} u^{+} & =0, & & \text { when } r>a, \text { (in vacuum) } \\
\Delta u^{-}+k^{2} \varepsilon_{d} u^{-} & =0, & & \text { when } r \leq a, \text { (in the dielectric) }, \\
\left.\left(u^{+}-u^{-}\right)\right|_{r=a}=\left.\frac{\partial}{\partial r}\left(u^{+}-u^{-}\right)\right|_{r=a} & =0, & & \text { (compatibility conditions) }
\end{aligned}
$$

In addition, $u^{+}$must be an outgoing wave at infinity. We use separation of variables. Substituting $u=R(r) \Theta(\theta)$ above and proceeding in the standard way, we conclude that

$$
\begin{aligned}
& u^{+}=H_{\tilde{n}}^{(1)}(k r)\left(\tilde{A} e^{i \tilde{n} \theta}+\tilde{B} e^{-i \tilde{n} \theta}\right) \quad \text { in vacuum, } \\
& u^{-}=J_{n}\left(k \sqrt{\varepsilon_{d}} r\right)\left(A e^{i n \theta}+B e^{-i n \theta}\right) n \quad \text { in the dielectric. }
\end{aligned}
$$

for some integers $n, \tilde{n}=0,1,2, \ldots$. Here we have picked solutions that are outgoing in the vacuum region and bounded inside the dielectric.

Now, the first transmission condition of (3.3) implies $n=\tilde{n}$,

$$
\tilde{A}=A \frac{J_{n}\left(k \sqrt{\varepsilon_{d}} a\right)}{H_{n}^{(1)}(k a)}, \quad \text { and } \quad \tilde{B}=B \frac{J_{n}\left(k \sqrt{\varepsilon_{d}} a\right)}{H_{n}^{(1)}(k a)},
$$

and the second condition of (3.3) further yields

$$
\left(R^{-}\right)^{\prime}(a) \Theta^{-}(\theta)=\left(R^{+}\right)^{\prime}(a) \Theta^{+}(\theta) .
$$

where the - and + signify the interior and exterior of the disk respectively. This implies that for each $n$, the values of $k$ must satisfy

$$
\sqrt{\varepsilon_{d}} J_{n}^{\prime}\left(k \sqrt{\varepsilon_{d}} a\right) H_{n}^{(1)}(k a)=\left(H_{n}^{(1)}\right)^{\prime}(k a) J_{n}\left(k \sqrt{\varepsilon_{d}} a\right) .
$$

We have not been able to analytically solve this equation for $k$. However, we can obtain numerical approximations to high precision by finding the roots of the function

$$
f_{n}=k\left(\sqrt{\varepsilon_{d}} J_{n}^{\prime}\left(k \sqrt{\varepsilon_{d}} a\right) H_{n}^{(1)}(k a)-\left(H_{n}^{(1)}\right)^{\prime}(k a) J_{n}\left(k \sqrt{\varepsilon_{d}} a\right)\right),
$$


TABLE 3.1

A few exact resonance values $k_{n, m}$

\begin{tabular}{|c|cccc|}
\hline$k_{n, m}$ & $n=0$ & $n=1$ & $n=2$ & $n=3$ \\
\hline$m=1$ & $0.436676-0.303945 i$ & $1.115540-0.239628 i$ & $1.756263-0.174352 i$ & $2.384047-0.121696 i$ \\
$m=2$ & $1.977701-0.279097 i$ & $2.716779-0.266504 i$ & $3.404368-0.245056 i$ & $4.064044-0.220085 i$ \\
$m=3$ & $3.542742-0.276273 i$ & $4.298557-0.271174 i$ & $5.013898-0.260996 i$ & $5.702569-0.248231 i$ \\
\hline
\end{tabular}

where we have multiplied by $k$ to remove a singularity.

We enumerate the exact resonance values of this problem as $k_{n, m}$, as for each $n$, we have a sequence of roots for (3.8), indexed by $m$. A few exact resonance values obtained for the case

$$
a=1 \quad \text { and } \quad \varepsilon_{d}=4
$$

are displayed in Table 3.1. Note that for $n>0$, each resonance value $k_{n, m}$ is of multiplicity two (both $A$ and $B$ in (3.5) are degrees of freedom), while for $n=0$ the resonance values $k_{0, m}$ are simple.

Now we report on some discrete approximations to these exact resonances for the disk. These approximations are computed using finite elements and PML. The exact problem can be cast as the eigenvalue problem of finding complex numbers $\lambda \equiv k^{2}$ and nontrivial eigenfunctions $u$ satisfying

$$
-\Delta u=\lambda \varepsilon(\boldsymbol{x}) u, \quad \text { on } \mathbb{R}^{2}, \quad \text { where } \varepsilon(\boldsymbol{x})=\left\{\begin{array}{l}
4, \text { if }|\boldsymbol{x}| \leq 1, \\
1, \text { if }|\boldsymbol{x}|>1,
\end{array}\right.
$$

with the additional condition that $u$ is an outgoing wave at infinity. (Note that the dielectric parameters are the same as that used to obtain Table 3.1.) We will use PML as an absorbing layer to exponentially damp the solution outside a fixed radius $r_{1}$, and then truncate the computational domain for some $r_{3} r_{1}$. In the truncated finite domain, we use the finite elements as the discretization method. This is a well known technique used for source problems $[1,2,3]$ with outgoing solutions, although its applicability to eigenvalue problems is less studied.

We first briefly describe the truncated PML and its finite element approximation. Our PML parameters are closer to [2,3] than the original ones of Berrenger [1]. In the region $r<1$, we set the actual coefficients given by our $\varepsilon$. The artificial coefficients forming PML are set outside radius $r=r_{1} \geq 1$. In the region $r_{1}<r<r_{2}$, we have a transitional variable coefficient, and in the region $r_{2}<r<r_{3}$ we have a constant artificial coefficient. Define

$$
\tilde{\sigma}(r)=\left\{\begin{array}{rl}
0, & \text { if } r<r_{1}, \\
\frac{s(r)}{s\left(r_{2}\right),} & \text { if } r_{1}<r<r_{2}, \\
1, & \text { if } r>r_{2},
\end{array} \quad \sigma(r)=\left\{\begin{aligned}
0, & \text { if } r<r_{1}, \\
\frac{d}{d r}(r \tilde{\sigma}(r)), & \text { if } r_{1}<r<r_{2}, \\
1, & \text { if } r>r_{2},
\end{aligned}\right.\right.
$$

where

$$
s(r)=\int_{r_{1}}^{r}\left(t-r_{1}\right)^{2}\left(t-r_{2}\right)^{2} d t .
$$




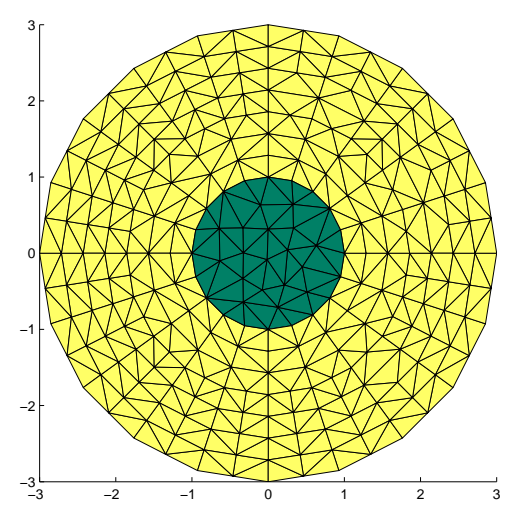

(a) The case $r_{3}=3$

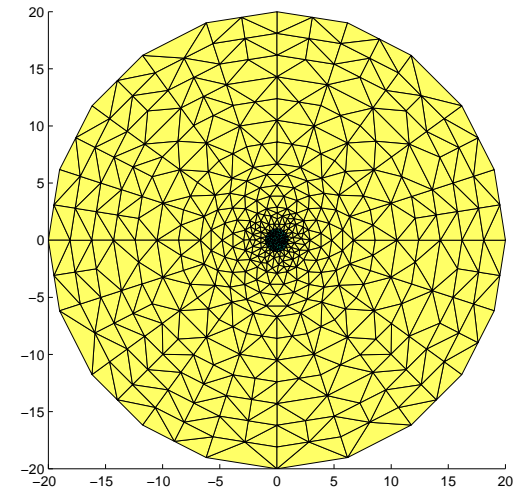

(b) The case $r_{3}=20$

FIG. 3.1. Finite element meshes (the dielectric is in darker shade)

Set $\gamma=1+i \sigma$ and $\tilde{\gamma}=1+i \tilde{\sigma}$. Then, with the coefficient matrices set to

$$
\mathcal{A}(\boldsymbol{x})=\frac{1}{r^{2}}\left(\begin{array}{ll}
\gamma x^{2}+\tilde{\gamma} y^{2} & x y(\gamma-\tilde{\gamma}) \\
x y(\gamma-\tilde{\gamma}) & \gamma y^{2}+\tilde{\gamma} x^{2}
\end{array}\right), \quad \mathcal{B}(\boldsymbol{x})=\varepsilon(\boldsymbol{x}) \gamma \tilde{\gamma}\left(\begin{array}{ll}
1 & 0 \\
0 & 1
\end{array}\right)
$$

the weak formulation of the truncated PML resonance problem is to find eigenvalues $\lambda \equiv k^{2}$ satisfying

$$
\langle\mathcal{A} \nabla u, \nabla v\rangle=\lambda\langle\mathcal{B} u, v\rangle, \quad \text { for all } v \in H_{0}^{1}\left(B_{r_{3}}\right),
$$

for some nontrivial eigenfunction $u$ in $H_{0}^{1}\left(B_{r_{3}}\right)$. Here $B_{r_{3}}=\left\{\boldsymbol{x} \in \mathbb{R}^{2}:|\boldsymbol{x}|<r_{3}\right\}$, and $\langle\cdot, \cdot\rangle$ denotes the $L^{2}\left(B_{r_{3}}\right)$ inner product. To discretize (3.10), we used Lagrange finite elements of degree one, on the meshes shown in Figure 3.1(a) and 3.1(b). The two meshes correspond to the two values of $r_{3}$ that we will investigate.

The discretization results in a large sparse generalized eigenvalue problem

$$
\mathrm{Ax}=\lambda \mathrm{Bx}
$$

where $\mathrm{A}_{i j}=\left\langle\mathcal{A} \nabla \phi_{j}, \nabla \phi_{j}\right\rangle, \mathrm{B}_{i j}=\left\langle\mathcal{B} \phi_{j}, \phi_{i}\right\rangle$, and $\phi_{i}$ 's are the usual nodal finite element basis. Note that this is a standard linear eigenvalue problem, because we have used PML coefficients that do not depend on frequency (unlike the ones in the original paper of Bérenger [1]). We shall see that in the asymptotic integral equation approach in the next subsection, we will get a nonlinear eigenproblem. Now, recall the exact resonant $k$ values calculated in Table 3.1. We compare the square roots of the eigenvalues computed by (3.11) with the exact values of Table 3.1.

The square root of the full spectrum of (3.11) in the case of the mesh in Figure 3.1(a) (the $r_{3}=3$ case), together with the first few exact resonance values are shown in Figure 3.2. As marked in the figure, a number of points in the computed spectrum lie far away from the exact resonances and must clearly be considered spurious.

Next, we systematically investigate the convergence of the first ten non-spurious resonance values to the exact value with respect to the discretization meshsize. Starting with the mesh in Figure 3.1(a), we perform a series of successive refinements. The 


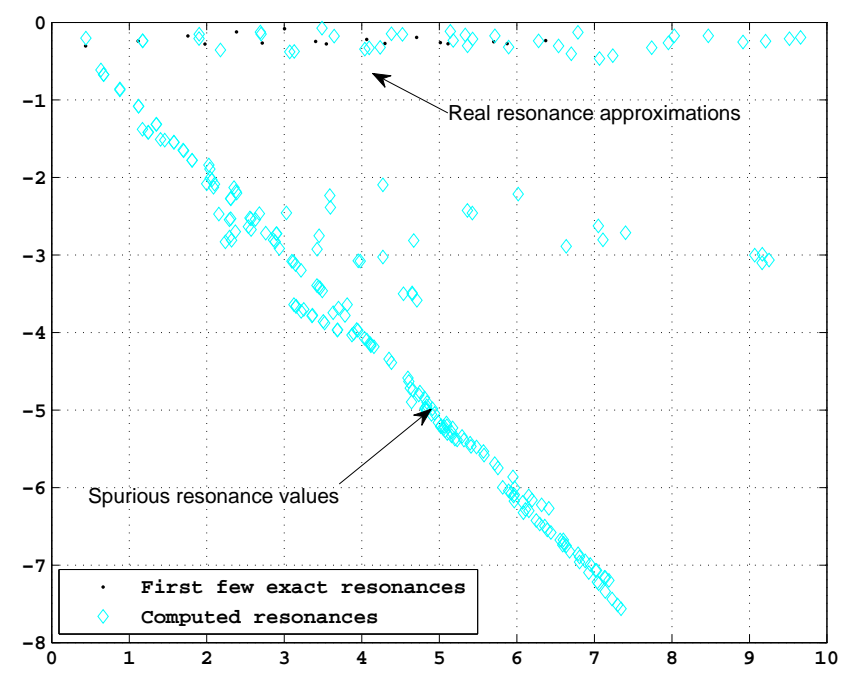

FIG. 3.2. Computed resonances for the case $r_{3}=3$

mesh at refinement level $J$ is obtained by joining the midpoint of the edges of each triangle of the previous level $J-1$. At each refinement, the coordinates of the newly created vertices on the dielectric-air interface are adjusted so that they lie exactly on the unit circle. Let us denote the by $k_{\ell}^{J}$ the $\ell$-th resonance value computed at refinement level $J$, where the ordering in $\ell$ is with respect to increasing real part, considering only the non-spurious values. The results are tabulated in Table 3.2. The definitions of mean orders of convergence in the table are as follows:

Apparent mean order of convergence for $\ell^{\text {th }}$ resonance $:=\frac{1}{3} \sum_{J=2}^{4} \log _{2}\left(\frac{\left|k_{\ell}^{J}-k_{\ell}^{J-1}\right|}{\left|k_{\ell}^{J}-k_{\ell}^{J+1}\right|}\right)$,

Actual mean order of convergence for $\ell^{\text {th }}$ resonance $:=\frac{1}{3} \sum_{J=2}^{4} \log _{2}\left(\frac{e_{\ell}^{J}}{e_{\ell}^{J+1}}\right)$,

where for each $\ell$, the true error $e_{\ell}^{J}$ is defined by $e_{\ell}^{J}=\left|k_{\ell}^{J}-k_{n, m}\right|$ for the $n, m$ values indicated in the first column of the table (under the title "Compare with $k_{n, m}$ "). The "apparent" rate quantifies the order of difference of approximations from successive refinements, and is a standard way to measure convergence rate in cases where we have no knowledge of the exact solution.

It is important to note the difference between the apparent and actual rates of convergence in the case of some resonance values (see rows with $\ell=1,2,3$ in Table 3.2). These suggest that although the computed resonances appear to converge at a second order rate, they converge to the wrong limit. We conjecture that this is due to the spectral changes caused by the truncation of PML at radius $r_{3}$. Consider the results in Table 3.3, where we report the resonance values obtained using the mesh in Figure 3.1(b), with $r_{3}=20$. Clearly there is a marked improvement in the actual convergence rates, supporting the conjecture that $r_{3}$ needs to be sufficiently large.

To summarize, we note the following difficulties encountered with the PML approach: 
TABLE 3.2

Apparent and actual convergence of computed resonance values

\begin{tabular}{|c|c|c|c|c|c|}
\hline \multicolumn{6}{|c|}{ Case $r_{3}=3$} \\
\hline \multirow{2}{*}{$\ell \backslash J$} & \multicolumn{5}{|c|}{ Computed resonance approximations $k_{\ell}^{J}$ (displayed up to 3 digits) } \\
\hline & Level 1 & Level 2 & Level 3 & Level 4 & Level 5 \\
\hline 1 & $0.441-0.202 i$ & $0.432-0.207 i$ & $0.430-0.208 i$ & $0.429-0.209 i$ & $0.429-0.209 i$ \\
\hline 2 & $1.173-0.239 i$ & $1.129-0.240 i$ & $1.118-0.241 i$ & $1.115-0.241 i$ & $1.114-0.241 i$ \\
\hline 3 & $1.175-0.236 i$ & $1.129-0.239 i$ & $1.118-0.241 i$ & $1.115-0.241 i$ & $1.114-0.241 i$ \\
\hline 4 & $1.901-0.209 i$ & $1.793-0.181 i$ & $1.765-0.176 i$ & $1.759-0.175 i$ & $1.757-0.174 i$ \\
\hline 5 & $1.902-0.151 i$ & $1.793-0.170 i$ & $1.766-0.173 i$ & $1.759-0.174 i$ & $1.757-0.174 i$ \\
\hline 6 & $2.176-0.356 i$ & $2.025-0.296 i$ & $1.990-0.283 i$ & $1.981-0.280 i$ & $1.978-0.279 i$ \\
\hline 7 & $2.692-0.125 i$ & $2.463-0.123 i$ & $2.404-0.122 i$ & $2.389-0.122 i$ & $2.385-0.122 i$ \\
\hline 8 & $2.700-0.150 i$ & $2.464-0.128 i$ & $2.404-0.123 i$ & $2.389-0.122 i$ & $2.385-0.122 i$ \\
\hline 9 & $3.070-0.380 i$ & $2.814-0.294 i$ & $2.742-0.273 i$ & $2.723-0.268 i$ & $2.718-0.267 i$ \\
\hline 10 & $3.128-0.374 i$ & $2.822-0.295 i$ & $2.744-0.274 i$ & $2.724-0.268 i$ & $2.719-0.267 i$ \\
\hline \multirow[b]{2}{*}{$\ell$} & \multirow{2}{*}{$\begin{array}{c}\text { Apparent mean order } \\
\text { of convergence }\end{array}$} & \\
\hline & & \multicolumn{4}{|c|}{$\begin{array}{l}\text { Difference between resonances from successive refinements } \\
\left|k_{\rho}^{1}-k_{\rho}^{2}\right| k_{\rho}^{2}-k_{\rho}^{3}\left|k_{\rho}^{3}-k_{\rho}^{4}\right|\end{array}$} \\
\hline 1 & \begin{tabular}{|c|}
1.90 \\
\end{tabular} & 0.0100 & 0.0029 & 0.0007 & 0.0002 \\
\hline 2 & 1.97 & 0.0440 & 0.0114 & 0.0029 & 0.0007 \\
\hline 3 & 1.96 & 0.0452 & 0.0118 & 0.0030 & 0.0008 \\
\hline 4 & 2.00 & 0.1119 & 0.0277 & 0.0070 & 0.0018 \\
\hline 5 & 1.99 & 0.1105 & 0.0276 & 0.0070 & 0.0018 \\
\hline 6 & 2.02 & 0.1628 & 0.0372 & 0.0096 & 0.0024 \\
\hline 7 & 1.97 & 0.2282 & 0.0592 & 0.0151 & 0.0038 \\
\hline 8 & 1.98 & 0.2372 & 0.0597 & 0.0153 & 0.0039 \\
\hline 9 & 1.92 & 0.2698 & 0.0744 & 0.0197 & 0.0050 \\
\hline 10 & 1.96 & 0.3160 & 0.0806 & 0.0212 & 0.0054 \\
\hline \multirow{2}{*}{$\begin{array}{l}\text { Compare } \\
\text { with } k_{n, m}\end{array}$} & \multirow{2}{*}{$\begin{array}{l}\text { Actual mean order } \\
\text { of convergence }\end{array}$} & \multicolumn{4}{|c|}{ Actual errors } \\
\hline & & $J=2$ & $J=3$ & $J=4$ & $J=5$ \\
\hline$\left|k_{1}^{J}-k_{0,1}\right|$ & 0.01 & 0.0971 & 0.0957 & 0.0954 & 0.0953 \\
\hline$\left|k_{2}^{J}-k_{1,1}\right|$ & 0.90 & 0.0134 & 0.0024 & 0.0015 & 0.0021 \\
\hline$\left|k_{3}^{J}-k_{1,1}\right|$ & 0.92 & 0.0139 & 0.0024 & 0.0015 & 0.0020 \\
\hline$\left|k_{4}^{J}-k_{2,1}\right|$ & 1.98 & 0.0370 & 0.0093 & 0.0024 & 0.0006 \\
\hline$\left|k_{5}^{J}-k_{2,1}\right|$ & 1.99 & 0.0370 & 0.0093 & 0.0023 & 0.0006 \\
\hline$\left|k_{6}^{J}-k_{0,2}\right|$ & 1.98 & 0.0501 & 0.0128 & 0.0033 & 0.0008 \\
\hline$\left|k_{7}^{J}-k_{3,1}\right|$ & 1.98 & 0.0793 & 0.0201 & 0.0051 & 0.0013 \\
\hline$\left|k_{8}^{J}-k_{3,1}\right|$ & 1.98 & 0.0802 & 0.0205 & 0.0052 & 0.0013 \\
\hline$\left|k_{9}^{J}-k_{1,2}\right|$ & 1.96 & 0.1009 & 0.0264 & 0.0067 & 0.0017 \\
\hline$\left|k_{10}^{J}-k_{1,2}\right|$ & 1.96 & 0.1090 & 0.0285 & 0.0073 & 0.0018 \\
\hline $\begin{array}{l}\text { Degrees of } \\
\text { freedom: }\end{array}$ & 233 & 969 & 3,953 & 15,969 & 64,193 \\
\hline
\end{tabular}

TABLE 3.3

Convergence when the larger domain is used, cf. Table 3.2.

\begin{tabular}{|c|ccccc|c|}
\hline \multicolumn{7}{|c|}{ Case $r_{3}=20$} \\
\hline Compare & \multicolumn{7}{|c|}{ Actual errors } & Mean order \\
with $k_{n, m}$ & $J=1$ & $J=2$ & $J=3$ & $J=4$ & $J=5$ & of convergence \\
\hline$\left|k_{1}^{J}-k_{0,1}\right|$ & 0.1096 & 0.0342 & 0.0105 & 0.0034 & 0.0020 & 1.44 \\
$\left|k_{2}^{J}-k_{1,1}\right|$ & 0.1283 & 0.0411 & 0.0122 & 0.0032 & 0.0008 & 1.83 \\
$\left|k_{3}^{J}-k_{1,1}\right|$ & 0.1299 & 0.0412 & 0.0122 & 0.0032 & 0.0008 & 1.83 \\
$\left|k_{4}^{J}-k_{2,1}\right|$ & 0.1763 & 0.0497 & 0.0140 & 0.0036 & 0.0009 & 1.90 \\
$\left|k_{5}^{J}-k_{2,1}\right|$ & 0.1961 & 0.0545 & 0.0155 & 0.0040 & 0.0010 & 1.90 \\
$\left|k_{6}^{J}-k_{0,2}\right|$ & 0.1376 & 0.0487 & 0.0130 & 0.0033 & 0.0008 & 1.84 \\
$\left|k_{7}^{J}-k_{3,1}\right|$ & 0.2965 & 0.0759 & 0.0201 & 0.0051 & 0.0013 & 1.97 \\
$\left|k_{8}^{J}-k_{3,1}\right|$ & 0.3089 & 0.0781 & 0.0205 & 0.0052 & 0.0013 & 1.97 \\
$\left|k_{9}^{J}-k_{1,2}\right|$ & 0.2237 & 0.0756 & 0.0182 & 0.0046 & 0.0012 & 1.89 \\
$\left|k_{10}^{J}-k_{1,2}\right|$ & 0.2229 & 0.0762 & 0.0186 & 0.0047 & 0.0012 & 1.89 \\
\hline Degrees of & \multicolumn{7}{c}{} \\
freedom: & 427 & 1,739 & 7,021 & 28,217 & 113,137 & \\
\hline
\end{tabular}


- It is necessary to separate the true eigenvalues from the spurious eigenvalues.

- Although eigenvalues may appear to converge, they can converge to the wrong value, if the domain is not large enough.

These problems were readily identified in this validation experiment because we know the exact solution. However, in a situation without any a priori knowledge of the exact solution, it is important to keep in mind that such difficulties can occur. For instance, in our experiments with PML in cases when exact solution is unknown (reported in later subsections), we needed to separate the true eigenvalues from the spurious eigenvalues. To identify the true eigenvalues, we used the following techniques: (i) We compared the variations in the computed spectrum when PML parameters were varied. (ii) We also compared the spectrum computed with the standard rectangular (tensor product type [3]) PML with the results from the circular PML in (3.9). The spectral points that persisted across these changes were considered to be the real eigenvalues. (We shall not report these details for brevity.) It is more difficult to overcome the discrepancy between the apparent and actual convergence. We typically experiment with an increasing set of $r_{3}$ values, holding meshsize (approximately) fixed, until the variation in the eigenvalues of interest becomes negligible. This often requires meshes with a large number of degrees of freedom and hence entails expensive computations.

3.2. A homogeneous thin membrane.. In this subsection, we will describe the integral equation approach to the computation of resonances, and compare the results from it to those obtained with PML. The resonating object is a thin homogeneous dielectric membrane occupying the rectangular region $[-0.5,0.5] \times[-h / 2, h / 2]$. The dielectric constant is set to the following function:

$$
\varepsilon(x, z)= \begin{cases}6 / h, & \text { if }|z|<h / 2, \quad x \in[-0.5,0.5] \\ 1, & \text { otherwise },\end{cases}
$$

i.e., we choose $\varepsilon_{0}(x) \equiv 6$. For our numerical experiments here, we choose a geometrically decreasing sequence of values for $h$.

Let us first describe the collocation discretization of the asymptotic integral equation derived in Section 2. The computational domain, which is now $[-0.5,0.5]$, is meshed by a grid of evenly spaced points set at a distance $\delta$ apart. Discrete approximations to resonance modes are now in the space $V_{\delta}$ of continuous functions which are linear in between adjacent grid points. Define the matrix valued function $\mathrm{S}: \mathbb{C} \mapsto \mathbb{C}^{N \times N}$ by $\mathrm{S}(\lambda)=\mathrm{I}-\lambda \mathrm{T}(\lambda)$, where I denotes the identity matrix, and the entries of the matrix $T(\lambda)$ are defined by

$$
[\mathrm{T}(\lambda)]_{i j}=-\int_{-1 / 2}^{1 / 2} \varepsilon_{0}\left(x^{\prime}\right) G_{\lambda}\left(x_{i}, 0, x^{\prime}, 0\right) \psi_{j}\left(x^{\prime}\right) d x^{\prime} .
$$

Here $\psi_{j}$ is the unique function in $V_{\delta}$ which is one at the $j$-th grid point and zero at all other grid points. With these notations, the discrete problem is the dense nonlinear eigenvalue problem of finding complex numbers $\lambda$ and corresponding nontrivial vectors $\mathrm{x}$ satisfying

$$
\mathrm{S}(\lambda) \mathrm{x}=0 .
$$

This can be rewritten as a nonlinear system for $\lambda$ and $\mathrm{x}$, to which Newton's method or its variants can be applied. To compute the matrix entries defined by (3.13) we split 
the integral into integrals over each mesh interval (of length $\delta$ ). On those intervals where the integrand is smooth, the integrals are approximated by high order Gaussian quadratures. We must be more careful in the intervals containing the singularity of $G_{\lambda}$. On such elements, we use an expansion of the integrand to approximate the integral. In all cases, our integral approximations are at least $O\left(\delta^{7}\right)$ accurate.

To solve (3.14), we use the residual inverse iteration analyzed by Neumaier [15]:

Algorithm 3.1 (Residual inverse iterations).

1. Input an initial approximation $\lambda_{0}$ close to the eigenvalue of interest. If $\mathrm{S}\left(\lambda_{0}\right)$ is invertible, continue.

2. Set (Wilkinson) initial guess $x_{0}$ for the eigenvector:

(a) Perform the QR-factorization $\mathrm{QR}=\mathrm{S}\left(\lambda_{0}\right)$.

(b) Let $\mathrm{b}=\mathrm{Q} \mathrm{n}$, where $\mathrm{n}$ is the vector whose components are 1 .

(c) Solve the linear system $S\left(\lambda_{0}\right) \tilde{x}_{0}=b$, by $\tilde{x}_{0}=R^{-1} n$.

(d) Normalize by $x_{0}=\tilde{x}_{0} / e^{*} \tilde{x}_{0}$, where $e$ is the unit vector with one in the position of the largest entry of $\tilde{x}_{0}$.

(e) Set $y^{*}=e^{*} R^{-1} Q^{*}$ for use later.

3 . For $l=0,1,2, \ldots$ (until a stopping criteria is met) do:

(a) $\lambda_{l+1}=\lambda_{l}-\frac{\mathrm{y}^{*} \mathrm{~S}\left(\lambda_{l}\right) \mathrm{x}_{l}}{\mathrm{y}^{*} \mathrm{~S}^{\prime}\left(\lambda_{l}\right) \mathrm{x}_{l}}$, where $\left[\mathrm{S}^{\prime}(z)\right]_{i j}=\frac{d}{d z}[\mathrm{~S}(z)]_{i j}$.

(b) $\tilde{\mathrm{x}}_{l+1}=\mathrm{x}_{l}-\mathrm{R}^{-1} \mathrm{Q} * \mathrm{~S}\left(\lambda_{l+1}\right) \mathrm{x}_{l}$

(c) Normalize by $x_{l+1}=\tilde{x}_{l+1} /\left\|\tilde{x}_{l+1}\right\|_{2}$.

These iterations can be stopped once $\left|\lambda_{l+1}-\lambda_{l}\right|$ is smaller than a prescribed tolerance. In step 3a of the algorithm, we have used one step of a one dimensional Newton iteration. We can substitute this step with multiple Newton iterations or other nonlinear solvers.

This algorithm works well in our application if good initial approximations $\lambda_{0}$ are given. In order to find good initial guesses, we borrowed a technique used for plotting the pseudospectra [18] of matrices. Namely, if $\sigma_{\min }(\mathrm{S})$ denotes the smallest singular value of $S$, then it is easy to see that

$$
\sigma_{\min }(\mathrm{S}(\lambda))<\delta \quad \text { if and only if }\left\|\mathrm{S}(\lambda)^{-1}\right\|_{2}>1 / \delta .
$$

Motivated by this, before launching the residual inverse iterations for fine meshes, we first use a coarse mesh to obtain an inexpensive matrix approximation $\mathrm{S}(\lambda)$. We then compute the minimum singular value $\sigma_{\min }(\mathrm{S}(\lambda))$ on a grid of $\lambda$ in the complex plane. For coarse meshes, $\mathrm{S}(\lambda)$ is a small matrix, so this computation is fast. Because of (3.15), the plot of the minimum singular values locates regions where the resolvent $\mathbf{S}(\lambda)$ is nearly singular, thus providing good initial guesses for Algorithm 3.1. For our current example of the homogeneous thin membrane, a coarse mesh resulting in a $20 \times 20$ matrix function $\mathrm{S}(\lambda)$ yields the contour plot of $\sigma_{\min }$ shown in Figure 3.3. Note that since $k=\sqrt{\lambda}$ is what we shall report, Figure 3.3 shows $\sigma_{\min }$ as a function of $k$ (rather than $\lambda$ ).

Next, we report the first few resonance values computed using Algorithm 3.1 applied to (3.14). We mesh the interval $[-0.5,0.5]$ uniformly with a mesh of meshsize $\delta_{0}=1 / 20$. To perform a study of convergence with respect to meshsize, we refine this coarse mesh, by splitting each grid element into two equal elements, so the meshsize at the refinement level $J$ is $\delta_{J}=2^{-(J-1)} / 20$. Denoting the $\ell$-th resonance value computed using the mesh at refinement level $J$ as $k_{\ell}^{J, \infty}$, the differences in the computed resonance values at successive refinements are are collected in Table 3.4. Examining these differences, we conjecture that the convergence rate of collocation discretization 


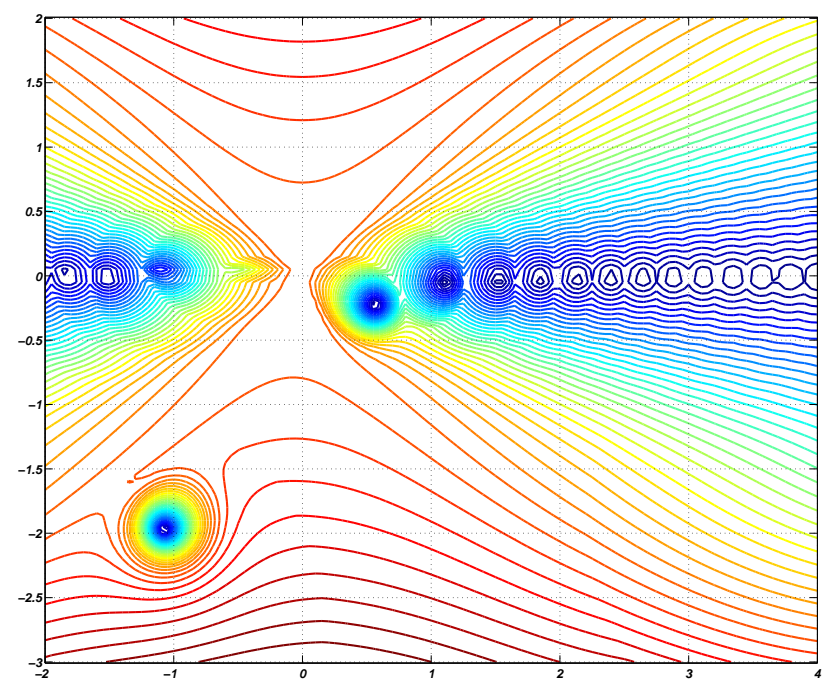

FIG. 3.3. Contour plot indicating the resonant frequencies in the $k$ plane.

TABLE 3.4

Resonance values (square roots $k=\sqrt{\lambda}$ ) from the collocation discretization of the asymptotic integral equation

\begin{tabular}{|c|cccccc|c|}
\hline$\ell$ & $\left|k_{\ell}^{1, \infty}-k_{\ell}^{2, \infty}\right|$ & $\left|k_{\ell}^{2, \infty}-k_{\ell}^{3, \infty}\right|$ & $\left|k_{\ell}^{3, \infty}-k_{\ell}^{4, \infty}\right|$ & $\left|k_{\ell}^{4, \infty}-k_{\ell}^{5, \infty}\right|$ & $\left|k_{\ell}^{5, \infty}-k_{\ell}^{6, \infty}\right|$ & $\left|k_{\ell}^{6, \infty}-k_{\ell}^{7, \infty}\right|$ & $k_{\ell}^{*, \infty}:=k_{\ell}^{7, \infty}$ \\
\hline 1 & 0.0002044 & 0.0000561 & 0.0000153 & 0.0000041 & 0.0000011 & 0.0000003 & $0.5650936-0.2220208 i$ \\
2 & 0.0020219 & 0.0005452 & 0.0001460 & 0.0000389 & 0.0000103 & 0.0000027 & $1.1080760-0.0456433 i$ \\
3 & 0.0072890 & 0.0019383 & 0.0005112 & 0.0001341 & 0.0000350 & 0.0000091 & $1.5159978-0.0284500 i$ \\
4 & 0.0163478 & 0.0043339 & 0.0011364 & 0.0002959 & 0.0000767 & 0.0000198 & $1.8255240-0.0175582 i$ \\
5 & 0.0303687 & 0.0080609 & 0.0021085 & 0.0005468 & 0.0001411 & 0.0000363 & $2.0959593-0.0137123 i$ \\
6 & 0.0490834 & 0.0130928 & 0.0034242 & 0.0008862 & 0.0002280 & 0.0000584 & $2.3307014-0.0110730 i$ \\
7 & 0.0733213 & 0.1801607 & 0.0073143 & 0.0018911 & 0.0004850 & 0.0001239 & $2.7440293-0.0080844 i$ \\
8 & 0.0964783 & 0.0377803 & 0.0099407 & 0.0025712 & 0.0006591 & 0.0001681 & $2.9294654-0.0065552 i$ \\
9 & 0.0523806 & 0.0493936 & 0.0130412 & 0.0033757 & 0.0008650 & 0.0002205 & $3.1026198-0.0063434 i$ \\
10 & 0.0090552 & 0.0628079 & 0.0166530 & 0.0043152 & 0.0011058 & 0.0002817 & $3.2674525-0.0052055 i$ \\
\hline
\end{tabular}

for the resonance values is $O\left(\delta^{2}\right)$ where $\delta$ is the meshsize. The last column of Table 3.4 lists the resonance values computed using the finest mesh.

We now compare these resonance values with those obtained using the PML approach. We enclose the dielectric in $[-0.5,0.5] \times[-h / 2, h / 2]$ by circles of radius $r_{1}, r_{2}$ and $r_{3}$ and set the PML parameters as described in $\S 3.1$. We experimented with a number of $r_{3}$ values and concluded that selecting $r_{3}=20$ seems appropriate to get good approximations in this example. We also needed to isolate the spurious eigenvalues from the interesting ones (see remarks at the end of $\S 3.1$ ). We shall consider the following geometrically decreasing sequence of membrane thicknesses:

$$
h=\frac{0.25}{2^{L-1}}, \quad L=1,2, \ldots, 7 .
$$

For each $L$ value, we mesh the domain $\left(B_{r_{3}}\right)$ such that mesh aligns with the dielectric boundaries. Furthermore, the meshes are such that for all values of $h$ considered, the dielectric region will always have four layers of elements. The meshsize inside the dielectric is thus maintained approximately at $h / 4$ by constraining the angles of the 
TABLE 3.5

Difference between the asymptotic and PML resonance approximations

\begin{tabular}{|c|ccccc|}
\hline$\ell$ & $\left|k_{\ell}^{\mathrm{pml}, 1}-k_{\ell}^{*, \infty}\right|$ & $\left|k_{\ell}^{\mathrm{pml}, 2}-k_{\ell}^{*, \infty}\right|$ & $\left|k_{\ell}^{\mathrm{pml}, 3}-k_{\ell}^{*, \infty}\right|$ & $\left|k_{\ell}^{\mathrm{pml}, 4}-k_{\ell}^{*, \infty}\right|$ & $\left|k_{\ell}^{\mathrm{pml}, 5}-k_{\ell}^{*, \infty}\right|$ \\
\hline 1 & 0.0411 & 0.0205 & 0.0101 & 0.0048 & 0.0021 \\
2 & 0.1655 & 0.0864 & 0.0444 & 0.0226 & 0.0115 \\
3 & 0.4086 & 0.2144 & 0.1102 & 0.0561 & 0.0284 \\
4 & 0.7001 & 0.3684 & 0.1896 & 0.0965 & 0.0488 \\
5 & 0.6427 & 0.5498 & 0.2841 & 0.1448 & 0.0733 \\
6 & 0.7790 & 0.7500 & 0.3884 & 0.1981 & 0.1003 \\
\hline
\end{tabular}

mesh triangles to never deteriorate below 25 degrees. With $\varepsilon(x, y)$ as in (3.12), we then solve the resulting finite element eigenproblem (3.11) for the first few eigenvalues and compare them with the resonance approximations previously displayed in the last column of Table 3.4.

One of our aims in this comparison is the verification of the theoretically predicted asymptotic convergence rate of $O(h)$ of Theorem 2.4. To realize this goal, we must avoid discretization errors as much as we can, but without going to prohibitively expensive meshsizes. Note that the first six resonance values in Table 3.4 have stabilized up to four digits at the seventh level of refinement, so we denote these six values by $k_{\ell}^{*, \infty}, \ell=1,2, \ldots, 6$, and use them for the comparison with the corresponding first six resonance approximations from PML. In order to avoid finite element discretization errors in the comparable PML resonance approximations, we perform multiple refinements of the finite element mesh until their first six resonance approximations have no variation in at least the first two significant digits. Denoting these approximations by $k_{\ell}^{\mathrm{pml}, L}$ for the case of membrane thickness $h=0.25 / 2^{L-1}$, we display in Table 3.5, the distance of these approximations to the asymptotic ones. The linear asymptotic convergence rate is clearly apparent.

Next we apply Corollary 2.5 to attempt to improve the asymptotic resonance approximations from the problem in the previous subsection. Recall that from the residual correction procedure described there, we have limiting resonance value $\lambda_{0}$ and a discrete approximation to the corresponding resonance function $u_{0}(x)$. We will apply these values to calculate

$$
\lambda_{0}+h \lambda_{1},
$$

to get what should be a better approximation to the resonance value $\lambda_{h}$ for a given total slab thickness $h$. Note that the correction

$$
\lambda_{1}=\lambda_{0}^{2} \frac{\left\langle g, u_{0}\right\rangle}{1+\lambda_{0}^{2}\left\langle D T_{0}\left(\lambda_{0}\right) u_{0}, u_{0}\right\rangle}
$$

where

$$
g(x)=-\int_{\Omega} G_{\lambda_{0}}\left(x, 0, x^{\prime}, 0\right) u_{0}\left(x^{\prime}\right) d x^{\prime}+\frac{\varepsilon_{0}(x) u_{0}(x)}{2}\left(\zeta^{2}+\frac{1}{4}\right),
$$

involves merely double integrations over $\Omega$, in this case a one dimensional domain. In the second term in the numerator the integration in $\zeta$ can be calculated exactly. The integral in the denominator is

$$
\left\langle D T_{0}\left(\lambda_{0}\right) u_{0}, u_{0}\right\rangle=-\int_{-.5}^{.5} \int_{-.5}^{.5} \frac{i \varepsilon_{0}\left(x^{\prime}\right)}{8 \sqrt{\lambda_{0}}} H_{1}^{(1)}\left(\sqrt{\lambda_{0}}\left|x-x^{\prime}\right|\right)\left|x-x^{\prime}\right| u_{0}\left(x^{\prime}\right) \overline{u_{0}}(x) d x^{\prime} d x .
$$



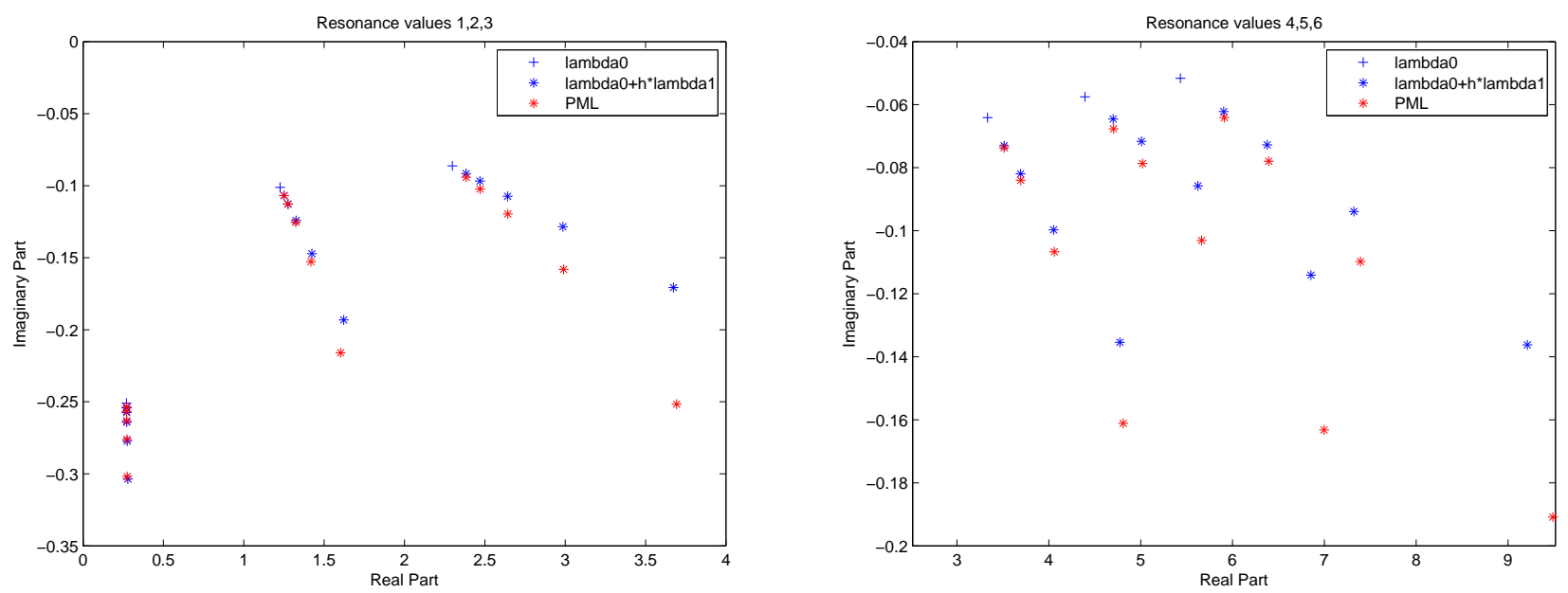

FIG. 3.4. The first six computed resonance values $\lambda$ in the complex plane for varying slab thickness.

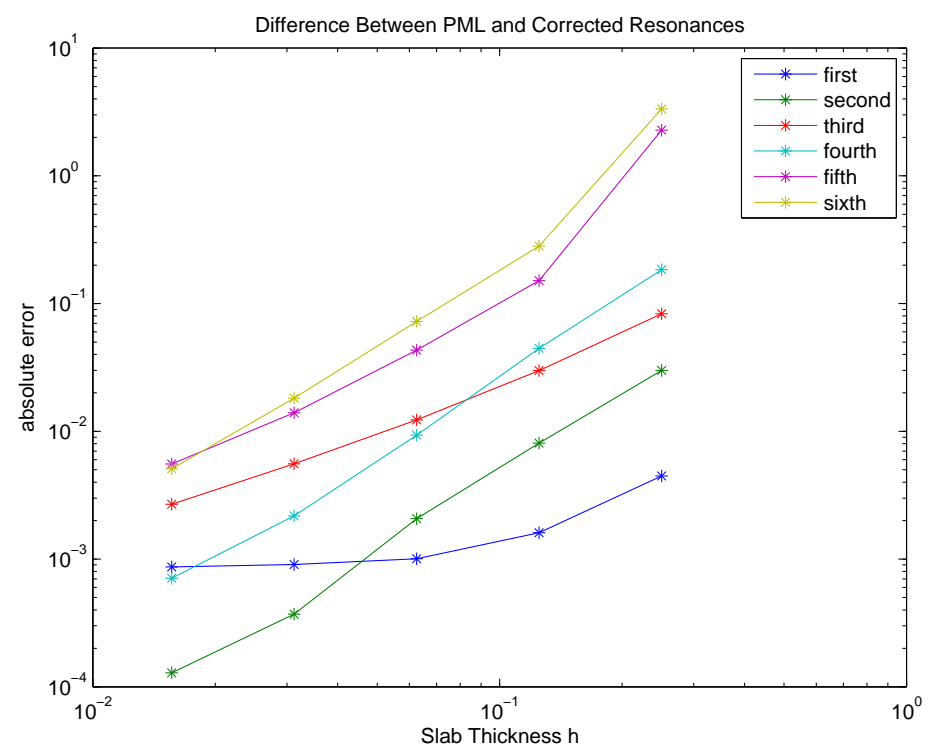

FIG. 3.5. Log-log plot of the absolute error between the PML computed and corrected asymptotic resonance values.

For both of the double integrals, we compute the inner integral using the piecewise linear basis functions for $u_{0}$, and for the outer integral we use the trapezoid rule. In all computations that follow the meshsize was $\delta=1 / 640$, the sixth refinement level, for which we believe the calculations of the first six limiting resonance values $\lambda_{0}$ are accurate up to about four significant digits. Recall that the PML values used from the previous section are accurate to about two significant digits.

Figure 3.4 shows all of the computed values plotted on the complex plane, and Figure 3.5 gives a log-log plot of the errors. For the first resonance, the corrected asymptotic values are within the presumed accuracy of the PML approximation for 


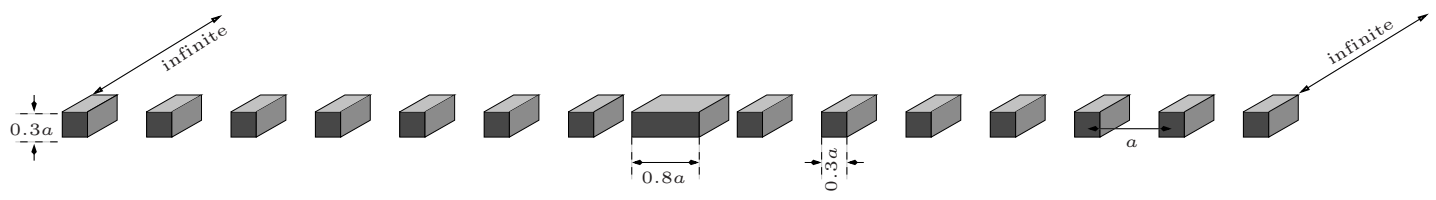

FIG. 3.6. The photonic dielectric structure

all values of $h$, hence we see the convergence flattening in the log-log plot. For the third resonance, the convergence appears only slightly more than linear, but all of the other values exhibit the significantly better than linear convergence expected from Corollary 2.5.

REMARK 3.1. The approximation from [14] used to obtain Corollary 2.5 deteriorates for larger frequencies, and we therefore expect that for the higher numbered resonances, the formula from Theorem 2.4 will be far superior. The computation of this more accurate correction will involve computing the application of the higher dimensional integral operator $T_{h}$, but will not require any inversion.

3.3. A photonic membrane.. In this subsection, we will describe the results of computation from a thin photonic membrane having a periodic dielectric pattern with a defect. The structure is shown in Figure 3.6 and was previously investigated in [5] by time domain methods. We will give results from both the PML and the asymptotic integral equation approaches. Unlike the previous subsections, our purpose here is not a convergence study, but rather a comparison with the results in [5]. The structure in Figure 3.6 is invariant in the third direction, so the model is reduced to one in the $x z$ plane perpendicular to the symmetry direction. Note that while fully periodic structures have band gaps, this structure is not periodic in that it has a defect in the center and has finite extent in the plane. Hence instead of defect eigenvalues, we seek resonances.

We choose the dielectric constant as in [5], namely, in the central defect column and the fourteen off-center columns, $\varepsilon(\boldsymbol{x})$ is 13 , while in the remaining region it equals one. We set the scaling parameter $a$ in Figure 3.6 to $1 / 14.3$ so that the $x z$ cross section fits into $[-0.5,0.5] \times[-h / 2, h / 2]$ with $h=0.3 a$. Since this $h$ is small, it is reasonable to attempt the asymptotic approach.

For the PML calculations, we enclose the $x z$ cross section of the photonic structure by disks of radius $r_{1}=0.6, r_{2}=2$ and $r_{3}=10$ and set the PML parameters as in the previous sections. This domain is meshed such that there are at least four layers of elements across the membrane thickness (and the mesh coarsen away from the dielectric). The computations then proceed similarly, except that to obtain higher accuracy, we now use Lagrange finite elements of degree five. The results are shown in Figure 3.8. The mode shown in Figure 3.8 $(a)$ is qualitatively similar to the one in [5, Fig. 5]. Furthermore, its corresponding resonance value is such that $k a / 2 \pi \approx 0.321$, a number close to the frequency of 0.313 reported in [5]. As seen from Figure 3.8(a), this mode is highly localized near the defect. Although there are many other resonances, as seen from Figure 3.8(d), localization near the defect or even near the membrane seems to be uncommon. (One other mode that is somewhat localized within the membrane is seen in the second plot of Figure 3.8(d).)

For the asymptotic approach, we set $\varepsilon_{0}=\varepsilon h$ with the $\varepsilon$ and $h$ as described above, and solve the resulting one dimensional nonlinear eigenproblem on $\Omega=[-0.5,0.5]$. The initial guesses for the nonlinear eigenvalue solver were obtained using the pseudospectrum- 


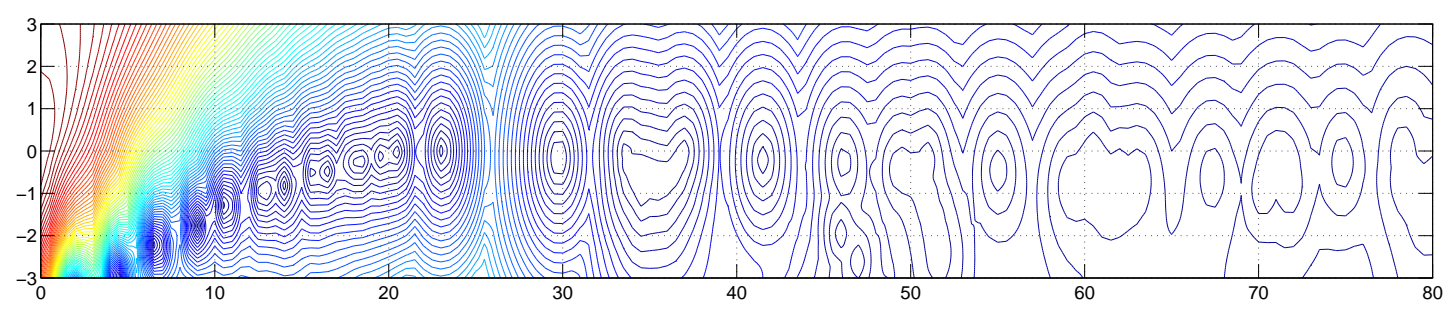

FIG. 3.7. Contour plot in the complex $k$ plane indicating the locations of the asymptotic resonances

$(c)$
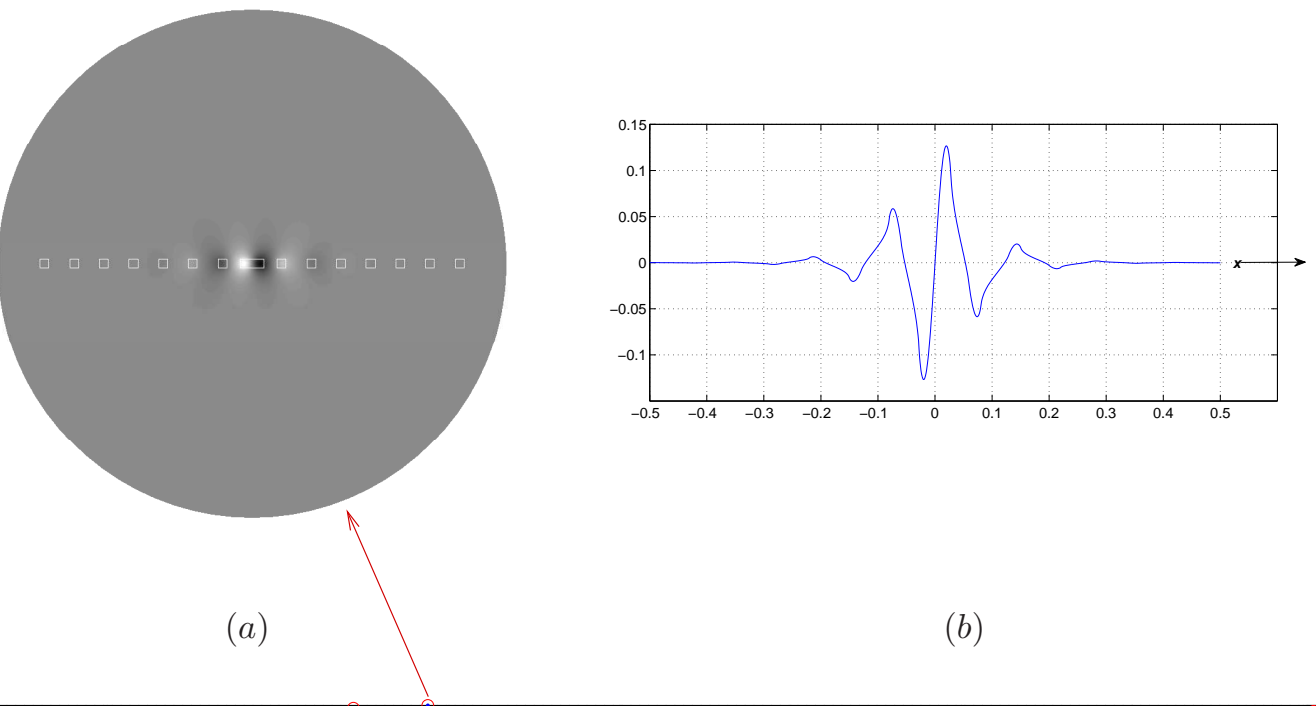

$(d)$

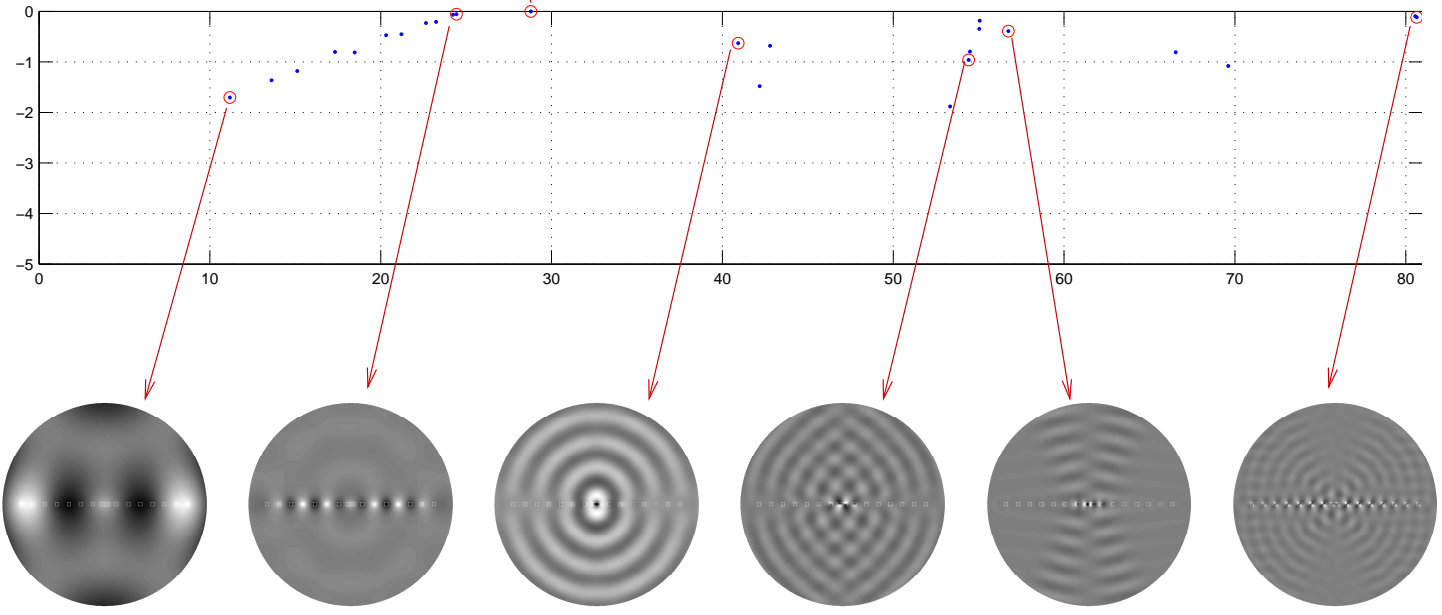

FIG. 3.8. A few resonances of the photonic membrane. (a) The highly localized resonance mode corresponding to $k \approx 28.7878-0.0017 i$ obtained using PML. (b) The corresponding mode obtained using the asymptotic approach, plotted on the limiting domain $[-0.5,0.5]$. (c) Point plot of a few resonant $k$ values computed using PML. (d) The modes corresponding to the $k$ values circled in $(c)$. (In all plots of the resonance modes, only the real part is plotted and only the region $r<r_{1}$ is shown.) 
TABLE 3.6

A few values of resonances $k$ for the photonic membrane

\begin{tabular}{|c|cc|}
\hline Values from PML & Asymptotic values & Corrected asymptotic values \\
\hline $20.3154-0.4704 i$ & $17.8719-0.2655 i$ & $19.9420-0.4049 i$ \\
$22.6512-0.2292 i$ & $19.7458-0.1116 i$ & $22.7624-0.1228 i$ \\
$24.2375-0.0658 i$ & $20.3675-0.0348 i$ & $23.7018-0.0493 i$ \\
$28.7878-0.0017 i$ & $23.0690-0.0006 i$ & $28.0236-0.0005 i$ \\
$40.9258-0.6283 i$ & $29.7120-0.1592 i$ & $39.1908-0.4074 i$ \\
\hline
\end{tabular}

like plot in Figure 3.7 (computed as described previously - see Figure 3.3). A few resonance values obtained using Algorithm 3.1 are reported in Table 3.6. The table also gives the corresponding resonance approximations from the PML calculations. There is good agreement between the PML and asymptotic values, especially after the correction. The most interesting mode is of course the one localized in the defect. The limiting, uncorrected, resonance value for this mode is such that $k a / 2 \pi \approx 0.257$, and produced the nonlinear eigenfunction in Figure 3.8(b). This qualitatively resembles not only the mode plot in [5, Fig. 5], but also the trace of the corresponding mode obtained from PML (Figure 3.8(a)) on the $x$-axis (the centerline of the dielectric). The corrected asymptotic resonance value is such that $k a / 2 \pi \approx 0.311$, very close to the value 0.313 reported in [5].

Considering that the PML eigenvalue problem we solved is of size $221201 \times 221201$, while the asymptotic problem is only of size $2289 \times 2289$, the advantages of the asymptotic approach are clearly evident for this particular geometry.

4. Discussion. We propose two methods for calculating resonance associated with the scalar wave equation. The first method is suited for thin, high index structures which are gaining popularity in the photonic bandgap community. It is an asymptotic method that exploits the specifics of the problem, and allows for the calculation of resonance to be carried out in one dimension less than the spatial dimension of the problem. The second method, based on the finite element method with the PML, is a general approach which is not restricted to thin structures. In this work, we examine the convergence properties of the finite element approach and used it to verify the approximation properties of the asymptotic method. A final set of calculations with both methods reproduce a photonic band gap resonance calculation reported in the literature.

For a thin membrane structure with high index, we find that the asymptotic method (2.3) is particularly effective. When discretized, it leads to a dense, but small, nonlinear eigenvalue problem. While we established approximation properties of the method, the numerical evidence is quite convincing. A higher order correction, which is easy to implement, provides more accuracy at a low cost. In comparison with the PML approach, the asymptotic method has the clear advantage of a smaller system, brought about by the dimensional reduction. Its disadvantage lie in the complications involved in solving a dense nonlinear eigenproblem.

The finite element PML approach is attractive because the matrices involved are sparse and the eigenvalue problem to find resonance is linear. It is also more widely applicable. One challenge in using this method is the presence of spurious modes. Our experience is that it is possible to identify spurious modes. Another unattractive feature of the PML approach is that the resonance values may appear to converge under refinement, but to incorrect limits. Our experience with this method, while 
limited, does gives us hope that it is possible to deal with these difficulties, and that it is possible to develop a robust finite element based method for calculating resonance. It remains to be seen however if it is a viable alternative to simple FDTD calculations.

Acknowledgments. The authors thank Joseph Pasciak for several enlightening discussions on exterior eigenvalue approximations.

\section{REFERENCES}

[1] J.-P. Berenger, A perfectly matched layer for the absorption of electromagnetic waves, J. Comput. Phys., 114 (1994), pp. 185-200.

[2] J. Bramble and J. Pasciak, Analysis of a finite element PML approximation for the three dimensional time-harmonic maxwell problem, Preprint, (2006).

[3] F. Collino And P. Monk, The perfectly matched layer in curvilinear coordinates, SIAM J. Sci. Comput., 19 (1998), pp. 2061-2090 (electronic).

[4] D. Colton and R. Kress, Inverse Acoustic and Electromagnetic Scattering Theory, vol. 93 of Applied Mathematical Sciences, Springer-Verlag, 1992.

[5] S. H. Fan, J. N. Winn, A. Devenyi, J. C. Chen, R. D. Meade, and J. D. Joannopoulos, Guided and defect modes in periodic dielectric waveguides, Journal of the Optical Society of America B-Optical Physics, 12 (1995), pp. 1267-1272.

[6] A. Figotin AND A. Klein, Localization of light in lossless inhomogeneous dielectrics, J. Opt. Soc. Am. A, 15 (1998), pp. 1423-1435.

[7] G. B. Folland, Introduction to Partial Differential Equations, Princeton University Press, 1976.

[8] R. Froese, personal communication.

[9] S. Hein, T. Hohage, W. Koch, And J. SchöBerl, Acoustic resonacnes in a high lift configuration, To appear in Journal of Fluid Mechanics, (2007).

[10] T. Kato, Perturbation theory for linear operators, Classics in Mathematics, Springer-Verlag, Berlin, 1995. Reprint of the 1980 edition.

[11] W. KocH, Acoustic resonances in rectangular open cavities, AIAA Journal, 43 (2005), pp. 23452349.

[12] K. D. Kоккотаs And B. G. Sсhмidt, Quasi-normal modes of stars and black holes, Living Rev. Relativ., 2 (1999), pp. 1999-2, 73 (electronic). http://www . livingreviews . org/lrr-1999-2.

[13] M. Lenoir, M. Vullierme-Ledard, and C. Hazard, Variational formulations for the determination of resonant states in scattering problems, SIAM J. Math. Anal., 23 (1992), pp. 579-608.

[14] S. Moskow, F. Santosa, And J. Zhang, An approximate method for scattering by thin structures, SIAM J. Appl. Math., 66 (2005), pp. 187-205.

[15] A. NeumaieR, Residual inverse iteration for the nonlinear eigenvalue problem, SIAM J. Numer. Anal., 22 (1985), pp. 914-923.

[16] J. E. OsBorn, Spectral approximation for compact operators, Math. Comp., 29 (1975), pp. 712725 .

[17] M. ReChBerger, Numerical methods for simulation of acoustic resonances, Master's thesis, Johannes Kepler Universität Linz, Altenberger Straße 69, Linz, Austria, November 2005.

[18] L. N. Trefethen, Pseudospectra of linear operators, SIAM Rev., 39 (1997), pp. 383-406.

[19] P. Villeneuve, S. Fan, S. Johnson, And J. JoAnnopoulos, Three-dimensional photon confinement in photonic crystals of low-dimensional periodicity, IEE Proc. Optoelectron., 145 (1998), pp. 384-390.

[20] J. Vuckovic, M. Loncar, H. Mabuchi, And A. Scherer, Optimization of the $q$ factor in photonic crystal microcavities, IEEE J. Quantum Elec., 38 (2002), pp. 850-856. 